\title{
A Simplified Model to Assess the Influence of the Configuration of Commercial Aircraft on Boarding and Deboarding
}

\author{
Diogo B. P. de Oliveira $\mathbb{D},{ }^{1}$ Jonathan N. Coelho $\mathbb{D},{ }^{1}$ and Alison de O. Moraes $\mathbb{D}^{2}$ \\ ${ }^{1}$ ITA, Praca Marechal Eduardo Gomes, 50-Vila das Acacias, Sao Jose dos Campos-SP-, Brazil 12228-900 \\ ${ }^{2}$ IAE, Praca Marechal Eduardo Gomes, 50-Vila das Acacias, Sao Jose dos Campos-SP-, Brazil 12228-900 \\ Correspondence should be addressed to Diogo B. P. de Oliveira; diogobpoliveira@gmail.com
}

Received 27 July 2020; Revised 12 January 2021; Accepted 19 January 2021; Published 9 February 2021

Academic Editor: Jacopo Serafini

Copyright ( 2021 Diogo B. P. de Oliveira et al. This is an open access article distributed under the Creative Commons Attribution License, which permits unrestricted use, distribution, and reproduction in any medium, provided the original work is properly cited.

\begin{abstract}
This paper presents an evaluation of the influence of aircraft configuration on the boarding and deboarding times using a simplified model and computer simulation. Boarding and deboarding times are important to airlines since both procedures are part of the critical path of the turnaround time (TAT) of aircraft in airports. During the TAT, a series of activities are performed in the aircraft in order to prepare it for the next flight. A reduction in boarding and deboarding times may represent a reduction in TAT for airlines. For the comparisons, three aircraft configurations are used: single aisle $3 \times 3$ ("six abreast"), single aisle $3 \times 2$ ("five abreast"), and single aisle $2 \times 2$ ("four abreast"), all with the same number of passengers. For the boarding analyses, two boarding procedures are used: random and random outside-in. The $2 \times 2$ aircraft interior configuration holds the shortest boarding times; deboarding times are similar for the three configurations. Also, a sensitivity analysis is carried out, and the results show that the higher the aircraft occupancy and the number of passengers with carry-on baggage, the higher are boarding and deboarding times, with the $2 \times 2$ having the lowest times in comparison with the other two configurations.
\end{abstract}

\section{Introduction}

Turnaround time (TAT) is the time taken for an aircraft in the airport to be prepared for the next flight. A series of activities take place in the TAT, such as boarding and deboarding of passengers, loading and unloading cargo, fueling, and catering [1]. Some of those activities can be performed simultaneously; however, a few of them are part of the critical path of TAT, such as boarding and deboarding of passengers.

Passenger boarding can be performed by queuing the passengers in a specific way at the gate. The way the passengers are queued to board the airplane is known as the boarding strategy. Each boarding strategy yields different results in terms of the boarding time. Boarding strategies are usually divided into random, by group and by seat [2]. In random boarding, all passengers are called together in one class [3] and board the airplane in no particular order, in a single block including all seats. This is different from the open seating method, in which passengers choose their seats inside the airplane. In the random method, passengers can board the airplane in no particular order but with an assigned seat prior to the start of the boarding process, while in open seating, the passengers choose their seats once they are inside the airplane. In outside-in boarding (a type of seat boarding), passengers seated in window seats board first, followed by middle seats, then finally aisle seats for both sides of the airplane. In outside-in boarding, passengers also have an assigned seat prior to the start of the boarding process. In this paper, random outside-in boarding is used, in which the order in terms of rows is not defined. Passenger deboarding, on the other hand, is performed with no special order. In this paper, since the model is a simplified one, passengers move in the airplane by occupying empty spaces in the aircraft aisle towards the exit door, and therefore, no special order is used.

During boarding and deboarding, passengers can block each other when they are performing activities in the airplane. Passengers stowing/retrieving luggage in the overhead bin block the passage in the aisle (aisle interference). Also, passengers that seat first in a row block the passage for a passenger that is seated closer to the window (seat interference). 
The main objective of airlines regarding the turnaround time is to perform all the activities as fast as they can in order to decrease the aircraft time on the ground but still considering all the safety involved in the processes. When it comes to the boarding procedure, the main objective of the airlines is to board the passengers in an optimal way to minimize the time required [2]. Another objective is to increase customer satisfaction and reduce the stress that passengers face during the boarding procedure.

Airlines, airports, and passengers have common interests in decreasing the boarding time for economic, operational, and customer satisfaction reasons, respectively. Different boarding strategies have been proposed in the literature with the objective of reducing the time of this activity, thus reducing aircraft time on the ground, making the aerial activity more profitable, improving the image of the airline, and allowing more aircraft to operate at the same airport.

Airlines generate revenue only when their planes are flying [4]. Regarding the increase of airplane time on the air, for scheduled flights, it depends on various factors such as the airline's fleet planning, schedule planning, passenger reservations, flight operations, ground operations, airplane maintenance system, air traffic controllers, and airport authorities [5]. For all these factors, there are improvement opportunities that can be explored by the airline. Since the present paper deals with airplane boarding and deboarding activities, all the analyses will focus on ground operations, especially boarding and deboarding procedures.

The airline market is a competitive business. An increase in fuel prices, maintenance and operation costs, and the competition for customer satisfaction lead airlines to make efforts in having the most efficient operation. Commercial airline management has been addressing the turnaround time because they believe it affects the overall success of the company. Nyquist and McFadden [6] suggest that for each minute the airplane is on the ground, it costs US\$30 to airlines. The boarding strategy combined with the airplane configuration could provide airlines a reduction in the TAT.

For $\mathrm{Wu}$ [1], airline operations at airports can be categorized by activities on the landside and on the airside of an airport. The activities on the airside are the ones described earlier included in the turnaround time of an aircraft. As for the landside, airline operations involve passenger checkin, baggage check-in, connecting passenger/baggage processing, cargo and goods handling, catering service preparation, and passenger boarding at the gates [1].

For all those operations, airlines have defined time constraints and standard operating procedures. For example, for each flight arriving or leaving the airport, the airline has an airport slot. An airport slot is "a permission given by a coordinator (airport coordination manager) for a planned operation to use the full range of airport infrastructure necessary to arrive or depart the airport on a specific date and time" (IATA 2017).

Although all the aforementioned activities on the landside and airside of airports have the potential for optimization and for that are worth studying, the present work deals specifically with the passenger deboarding and boarding activities. Passenger deboarding is accomplished by all the passengers getting off their seats and leaving the airplane at the same time. Usually, airlines do not exercise control over this process, and all the passengers deboard in no specific order or sequence. However, it is possible for airlines to control the sequence on passenger boarding, which can represent reducing the TAT.

A good overview of aircraft airport operations can be found in [7]. The study presents an introduction to ground operations focusing on the aircraft turnaround and passenger process. Also, the study analyzes current challenges for aircraft operators, such as airport capacity constraints, schedule disruptions, and the increasing cost pressure (the pressure on airlines to become more competitive). Then, a review of existing modeling and simulation frameworks for aircraft ground operation and ground operation research projects (concept studies) is presented. The conclusion is that the optimized use of airport infrastructure and the reduction of additional buffers allow faster travels for passengers and that passengers and airlines could benefit from shorter boarding procedures.

Different authors in the literature aimed to develop optimal boarding strategies in order to reduce passenger boarding times $[4,8,9]$. The authors use different approaches in order to evaluate the boarding problem.

Bazargan [4] called his model a mixed-integer linear program, in which its objective function has a goal to minimize the total expected seat and aisle interferences. A good number of boarding strategies were evaluated, and a simulation model was used to analyze the interference of the speed of boarding the passengers in the boarding patterns. The author recommends different variants of the reverse pyramid as boarding strategies since they "provide a lower number of interferences than other strategies as well as accommodating neighboring passengers to board together" [4].

The Steffen Method is an optimal boarding method developed by Jason Steffen [8] that allows multiple passengers to load their luggage at the same time. Steffen [8] used the Markov Chain Monte Carlo (MCMC) algorithm to find the optimal loading order. The objective of the simulation was to find the configuration that allows the maximum number of passengers to stow their luggage in the overhead bins at all times during the boarding procedure. The author explains that the Steffen Method is difficult to perform and proposes a modified optimal method that consists of passengers being queued in seats separated by two rows of one side of the aisle. He then compares its optimal boarding strategy with backto-front, outside-in, Steffen Method, and modified optimal method. Back-to-front had the worst performance, while the Steffen Method performed the best.

Similar to Steffen [8], Milne and Kelly [9] presented an optimal boarding method that minimizes the total time to board the airplane. The difference between the two methods is that the Steffen Method assigns passengers to a specific numerical position in line that depends on their seat location [8] while the method from [9] assigns airplane passengers to a seat based on the number of carry-on luggage they have so that the luggage is distributed evenly throughout the plane. The procedure for boarding in [9] consists of four steps described as follows: (1) assign sets of carry-on bags to rows; 
(2) within each row, assign sets of carry-on bags to seats; (3) assign passengers to seats matching the carry-on baggage assignments from step (2); and (4) passengers board according to the Steffen sequence based on their assigned seats.

Milne and Kelly [9] compared their boarding strategy to the Steffen Method and concluded that their method is about 1 to $3 \%$ faster than the one proposed in [8] in terms of the total time. The authors stated that both methods can be implemented by airlines and that the information of the amount of luggage carried by passengers can be obtained, with the industry moving in this direction. Milne and Kelly [9] concluded that their method is feasible and could provide savings to airlines.

In contrast to those studies aforementioned, Ferrari and Nagel [10] focus on the robustness of the strategies under disturbances to the boarding process, such as a certain number of passengers not following their boarding group (but boarding earlier or later), aircraft dimensions, and occupancy level of the airplane.

The authors performed a sensitivity study, known as the "average worst case" boarding model [10], to evaluate the aforementioned disturbances. They concluded that as for aircraft dimensions, in order to have a robust boarding process for different layouts, only strategies that consider boarding by seat, seat group, and pyramid are recommended. In the seat group strategy, seating is divided horizontally into a specified number of groups and horizontally by columns, with the boarding groups coming from back to front and from outside to inside, while the pyramid strategy is the one achieved by merging the seat group strategy diagonally [10]. For the early/late passenger effect, Ferrari and Nagel [10] state that for occupancy levels greater than 50\%, surprisingly, the backto-front strategies (boarding passengers from the back of the aircraft to the front) reduce their boarding times when passengers disobeyed their assigned boarding group. The authors recommend boarding by column and seat group strategies, as they offer good stability under the disturbances considered with good performance.

In a recent paper, Zeineddine [11] proposes an optimal boarding strategy called Dynamically Optimized Boarding (DOB). The authors claimed that previous optimal strategies did not account for the aspect of allowing families and groups to board together in cliques. Zeineddine [11] then stated that the objective of the proposed method is to reduce boarding time in an optimal procedure that reduces seat and aisle interferences and allows passengers' cliques to board together.

Zeineddine [11] compared their method to random boarding, back-to-front, window-middle-aisle, Steffen Method [8], and Sorted-Boarding-Groups (SBG). The DOB strategy had the closest performance to the Steffen Method but had a longer boarding time. The authors emphasized that the Steffen Method did not account for passengers traveling in groups (cliques) and concluded that DOB should be preferred by airlines in order to reduce boarding time since it "satisfies the passenger's crucial need to board in cliques and it ensures the seating of passengers in their preferred seats with minimum boarding interferences" [11].

After reviewing the most expressive studies on the passenger boarding problem, some common factors can be con- sidered. The first factor is the study approach, which could be field studies, computer simulation, or analytical models. The second is that the majority of the authors focused on singleaisle, short-haul airplanes for their analyses. The third factor is the importance of the carry-on luggage in the boarding process, as the process of stowing the luggage in the overhead bins could lead to passenger interferences, which increase the boarding time. The fourth factor is the psychological aspects and behavior of passengers, which is presented by recent studies such as Kierzkowski and Kisiel [12] and Schultz [13].

Based on the above, the main objective of this paper is to analyze the influence of the aircraft interior seating configuration on the boarding and deboarding times. A series of computer simulations are presented with two boarding strategies: random boarding and random outside-in boarding. Three aircraft configurations are analyzed: single aisle $3 \times 3$ ("six abreast"), single aisle $3 \times 2$ ("five abreast"), and single aisle $2 \times 2$ ("four abreast"), all with 120 passengers. After that, a sensitivity analysis is presented to evaluate the influence of the aircraft occupancy and the number of passengers with carry-on baggage on boarding and deboarding. It is shown that for all boarding procedures, the $2 \times 2$ interior configuration is the one with the shortest times, followed by $3 \times 2$ and $3 \times 3$. Different from passenger boarding, the deboarding times for all configurations are very similar, indicating the aircraft configuration does not exert a great influence on the deboarding process. Since the $2 \times 2$ is the one that holds the shortest boarding times and deboarding times are similar amongst the configurations, the $2 \times 2$ configuration is the one with the shortest TAT, and consequently, the greatest potential for cost savings for airlines.

The present work is divided into five sections. The first section presents an introduction to the turnaround activities and the boarding strategies used by airlines, the problem description studied in this work, and the motivation for this work. Also, a historical overview of the literature with the main contributions to the subject is presented. The second section describes the model used in this work for the simulations, as well as all the constraints and assumptions considered for the analysis. The third section presents the results for all the simulations performed for passenger deboarding and boarding for three aircraft with the same number of passengers and having the configurations of single aisle $3 \times 3$ ("six abreast"), single aisle $3 \times 2$ ("five abreast"), and single aisle $2 \times 2$ ("four abreast"), using random and random outside-in boarding methods. The fourth section presents a sensitivity analysis of the model presented in the third section, considering different disturbances in the boarding process. The fifth section presents the conclusions of the present work, with the main results, analysis, assumptions, and the limitations of the model simulations. Also, opportunities for future studies are highlighted.

\section{Model Description}

The computer model used in this paper represents the interactions that passengers have during the boarding and deboarding processes. For those interactions, two classes are defined: passenger and airplane. The tool used for the 


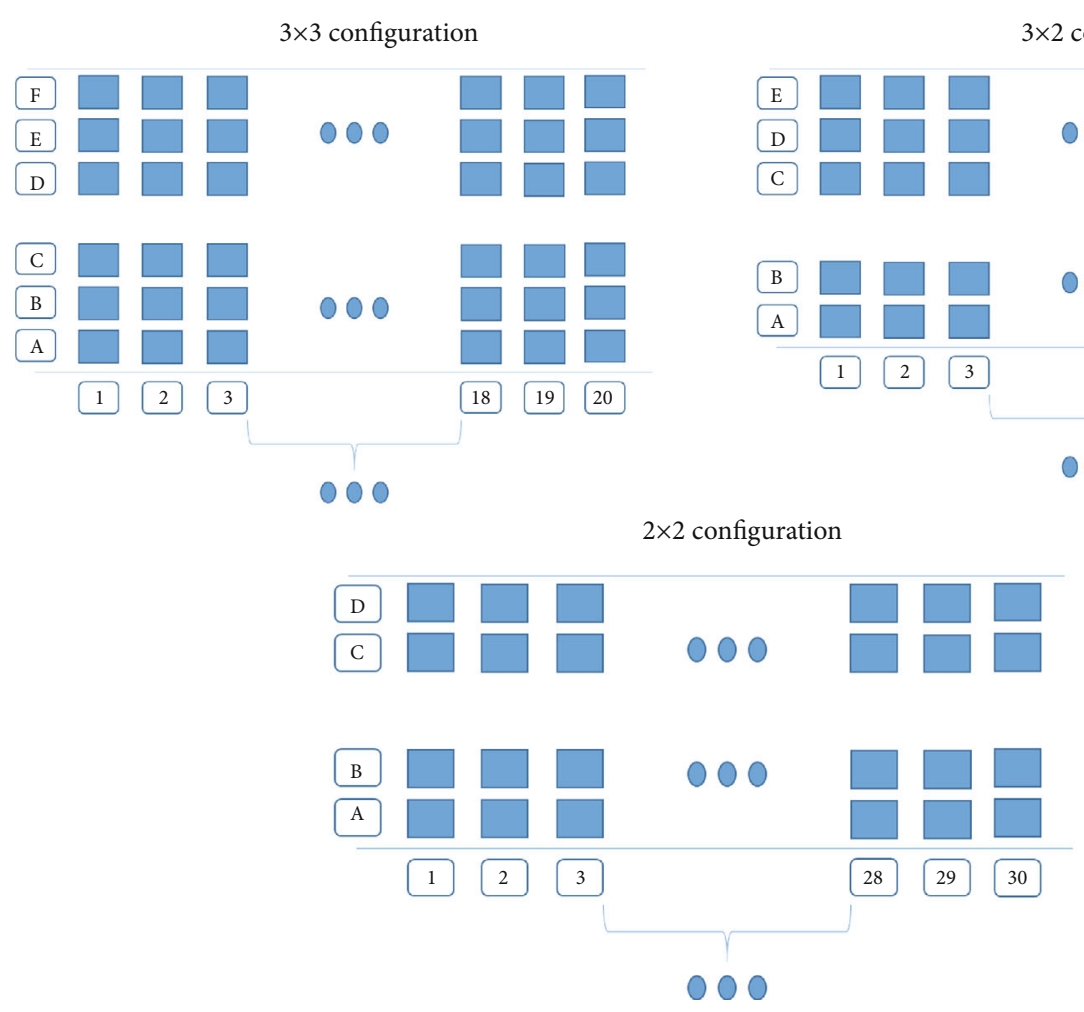

FIgURE 1: Aircraft configuration for case studies.

simulation was developed in MATLAB ${ }^{\mathrm{TM}}$. The computer model is based on object-oriented programming, in which the boarding and deboarding processes of an airplane are defined within the model with different inputs and outputs.

The passenger class presents all characteristics from the passengers, such as present position, assigned seat, time to execute actions, and seating status. Since this is a simplified model, all the passengers have the same average characteristics and no special model of their movement in the aircraft is adopted. Passengers perform one step at each time step in the simulation, with each time step having 1 second. In terms of passengers walking down the aisle, it is assumed that it takes one time step for the passengers to pass each row. The main characteristics that are defined within the model are the time a passenger takes to sit down, stow/retrieve baggage, and get up. During the sit down process, other passengers in the same row have to wait for the passenger that is sitting down to execute this action. The same is valid for getting up. As for the stow/retrieve baggage process, the passengers in the aisle behind the passengers performing these actions cannot move until the action is finished.

Some of the properties mentioned above are defined within the computer model and are updated depending on each passenger and simulation (time of next action, seat position, and seating status). Nonetheless, some of them depend on external parameters (time to sit down, time to get up, time to stow/retrieve carry-on baggage, and carry-on baggage). Those parameters are used to define the passenger and are set up by the model user before the simulation starts as inputs.

The airplane class, on the other hand, presents all the characteristics from the aircraft itself (such as interior config- uration, seats per row, and total number of seats) and for the boarding and deboarding procedures (such as boarding strategy, number of passengers, and boarding and deboarding times). This class represents the aircraft interior and the boarding and deboarding procedures that are executed by the passengers. It is the main part of the computer model since all the tasks and activities are taking place. For this paper, all the activities that take place outside the aircraft are not considered, which means that as long as the passengers are available to execute the actions inside the aircraft, it does not matter what happens before they are in (boarding) or after they are out (deboarding).

Similar to the passenger class, some of the properties mentioned above are defined within the computer model and are updated depending on each passenger and simulation (boarding time, deboarding time, and interior). Some of them depend on external parameters (number of passengers, number of aisles, number of rows, and number of passengers per row). Those parameters are used to define the aircraft and are set up by the user before the simulation starts as inputs.

The aircraft configurations considered in this paper are as follows: single aisle $3 \times 3$ ("six abreast"), single aisle $3 \times 2$ ("five abreast"), and single aisle $2 \times 2$ ("four abreast"), all with 120 passengers, as shown in Figure 1. Since the number of passengers is the same for the three configurations, the number of rows is different amongst configurations to accommodate the passengers. The single aisle $3 \times 3$ has 20 rows, the single aisle $3 \times 2$ has 24 rows, and the single aisle $2 \times 2$ has 30 rows. This choice is based on the fact that those configurations usually are used in regional short-haul flights that usually perform back-to-back flights during the day. Therefore, 
reducing the TAT for all flights for an aircraft may represent an extra flight during the day, which may result in extra income for the airlines. This outcome would be added to the reduction of costs by airlines in airport operations.

Boarding and deboarding procedures are represented in the model as a set of tasks for the passengers who have two objectives: to sit down in the boarding and to leave the airplane in the deboarding. All the interferences that happen in the passengers' way to their objectives are defined as constraints in the model. Both procedures use only the front door of the aircraft.

The boarding process is considered a network of tasks with precedences and constraints. The airplane boarding consists of one task per passenger, which is to sit down. The precedence relations or constraints are the conflicts that may appear during the passenger path to his/her assigned seat and the obligation of stowing his/her carry-on luggage in the overhead bin. For example, a given passenger may be blocking the aisle stowing his/her carry-on luggage, and while this activity is taking place, other passengers who are behind the given passenger cannot proceed to their assigned rows until he/she clears the aisle. During the boarding process, passengers enter the aircraft using the front door and queue in a single line walking down the aisle until they reach the row of their assigned seat. The passenger then puts his/her luggage in the overhead bin and finally sits down.

For this paper, two boarding strategies are considered: random boarding and random outside-in boarding. In the random outside-in boarding, passengers are aligned in the regular outside-in way, first the window seat group, then the middle seat group, and then the aisle seat group. Then, the groups are mixed randomly but maintaining the window-middle-aisle order. In order to control the boarding sequence, call-off systems are needed. In typical boarding procedures, gate agents announce which boarding group is allowed to board. This is how passengers are queued prior to boarding the aircraft. Call-off systems are not thoroughly analyzed in this paper, and it is assumed the airline will follow its own system considering the boarding strategy defined.

For the simulation, the boarding process is designed as a temporal loop that considers each passenger at a time and consists of three main steps:

(1) Firstly, it is checked if the passenger can execute an action, taking into consideration the precedence of tasks mentioned before; if the passenger cannot execute an action, the program goes to the next passenger; otherwise, it is checked if the passenger is already seated or not

(2) Secondly, if the passenger is not seated, his/her position is updated in the program. If the passenger is not seated, it is checked if he/she is located in the position of the right row where the seat assigned to $\mathrm{him} / \mathrm{her}$ belongs. If he/she is not located in the right row, it is checked if the passenger can walk, considering the precedence tasks mentioned before (aisle interference)
(3) Thirdly, if the passenger is located in the right row, he/she stows the carry-on luggage (if he/she possesses one) and checks if there is a passenger blocking the seat (seat interference). If so, the blocking passenger needs to get up in order to allow the passage of the current passenger. After the current passenger and blocking passenger sit down, the program goes to the next passenger and begins Step 1 again

For the boarding procedure described above, the computer model considers a time step of one second for each round of the temporal loop. This means that actions are only changed (if allowed to) at every second. Therefore, every temporal loop adds one second to the total time and the simulation ends when all the passengers have been seated.

Similar to the boarding process, the deboarding process is considered a network of tasks with precedences and constraints. The airplane deboarding consists of one task per passenger, which is to get off the airplane. The precedence relations or constraints are the conflicts that may appear during the passenger path to the airplane door and consequently out of the airplane.

During the deboarding process, passengers get up from their seats, retrieve their carry-on luggage (if he/she possesses one), and then walk down the aisle until they reach the airplane door, where they can finally deboard the airplane. For this paper, the deboarding process is considered from the moment the first passenger gets up until the moment the last passenger leaves the airplane. Different from the boarding procedure, the deboarding process does not take into account any special queuing or passenger sequence. Passengers deboard the airplane by occupying empty spaces. In terms of sequence within one row, passengers from the closest seats to the aisle deboard first. While the passengers closer to the aisle are performing the getting up and retrieving baggage actions, the passengers with the same row closer to the window have to wait, and they will just occupy the empty space when the first passengers move further in the deboarding process. In this model, human behavior, such as passenger eagerness to get out of the airplane during the deboarding process, is not taken into account.

Similar to the boarding process, the deboarding process is designed as a temporal loop that considers each passenger at a time and consists of three main steps:

(1) Firstly, it is checked if the passenger can execute an action, taking into consideration the precedence of tasks mentioned before; if the passenger cannot execute an action, the program goes to the next passenger; otherwise, it is checked if the passenger is still seated or not. If the passenger is not seated, then it is checked if he/she has left the plane. If this passenger is still on the plane, he/she waits or walks, depending if there is another passenger blocking the way or not

(2) Secondly, if the passenger is still seated, it is checked if he/she is seating in a middle or window seat. If this is positive, then it is checked if the passenger seating next has left the seat. If this is negative, then the current passenger waits 
TABLE 1: Validation procedure results.

\begin{tabular}{lccccc}
\hline Round & Aircraft model & Procedure & A1 & $\begin{array}{c}\text { Simulation time (min:sec) } \\
\text { 95\% confidence }\end{array}$ & Measured time (min:sec) \\
\hline 1 & 174 passengers & Boarding & {$\left[\begin{array}{lll}3 & 3 & 5\end{array}\right]$} & $15: 30$ & $15: 00$ \\
3 & 174 passengers & Deboarding & {$\left[\begin{array}{llll}3 & 3 & 5\end{array}\right]$} & $8: 21$ & $8: 00$ \\
4 & 228 passengers & Boarding & {$\left[\begin{array}{lll}3 & 3 & 5\end{array}\right]$} & $19: 54$ & $19: 00$ \\
5 & 228 passengers & Deboarding & {$\left[\begin{array}{lll}3 & 3 & 5\end{array}\right]$} & $10: 25$ & $10: 00$ \\
\hline
\end{tabular}

(3) Thirdly, if the current passenger can leave his/her seat, it is checked if the aisle is free and also if there is a passenger close to that position. If this is positive, there is a $95 \%$ chance that the current passenger will wait for that approaching passenger to walk by until he/she may enter the aisle. If the current passenger has carry-on luggage, he/she retrieves it from the overhead bin and gets up to the aisle. If not, he/she just gets up to the aisle. After the current passenger gets up, the program goes to the next passenger and begins Step 1 again

Similar to the boarding procedure, for the deboarding procedure described above, the computer model considers a time step of one second for each round of the temporal loop. Therefore, every temporal loop adds one second to the total time and the simulation ends when all the passengers have left the airplane.

\section{Boarding and Deboarding Analyses}

The purpose of the current section is to present a discussion of the simulations and analyses performed using the model described in Section 2. The first subsection describes the validation process that was performed in order to validate the computer model. The second subsection presents the results and discussions for the boarding analysis. The third subsection presents results and discussions for the deboarding analysis, and the fourth subsection presents an analysis combining boarding and deboarding for the three configurations.

For all analyses in this paper, 500 simulations were performed, and a histogram was created for all simulations. The histograms were then transformed into fitted normal distribution curves since they held similar shapes and also in order to make the comparisons between simulations easier. For all analyses, two confidence levels are used for the results: 95\% and $50 \%$. Here, a confidence level is defined as the probability in which the result is shorter than the defined value. This means that only $(100-$ confidencelevel $) \%$ of the results in the distribution will be greater than the informed value.

3.1. Model Validation. The first analysis of the model described in Section 2 was to perform its validation and assure that it holds close results to real boarding and deboarding processes. In order to do that, data was collected for boarding and deboarding times for $3 \times 3$ aircraft configuration with 174 passengers and 228 passengers of a major South American airline, collected in Guarulhos International Airport (Sao Paulo, Brazil) on August 27, 2018.
For the validation of the computer model, a series of simulations for the boarding and deboarding processes were conducted in order to assess the parameters for the passenger action times (get up, sit down, and stow/retrieve baggage). For each round of the simulation, the action times were changed in order to approach the best solution. For each round, 100 simulations of the boarding process were carried out. After that, a 95\% confidence level was used for the time distribution, and a boarding time and deboarding time were defined for each round.

The following assumptions were used for the validations process:

(i) One carry-on baggage per passenger

(ii) One class for the airplane configuration

(iii) Boarding strategy of back-to-front with 3 groups (similar boarding procedure to the one executed)

(iv) Airplane at full capacity (100\% load factor)

The parameters to be validated were passenger action times: get up, sit down, and stow/retrieve baggage. The approach used for all the rounds of simulations was trial and error based on empirical and experience data. For the validation process, first, the 174-passenger aircraft configuration was used as the aircraft model. After a reasonable match result for the boarding time for the $3 \times 3$ aircraft configuration was reached, the vector for the action time for the passengers was then used in other simulations with the 228-passenger aircraft configuration to test the consistency of the results using the action time found. The validation results are shown in Table 1 , where $A 1$ is the $1 \times 3$ vector in which each term represents the time spent for each passenger to sit down, to get up, and to retrieve/stow carry-on baggage, respectively.

The tuning parameters for passenger action times are the same for boarding and deboarding procedures. As a result of the validation process, the action times that held the closest results and therefore are the ones that will be used are as follows:

(i) Time to sit down is 3 seconds

(ii) Time to get up is 3 seconds

(iii) Time to stow/retrieve baggage is 5 seconds

Although the simulation results are close to the measured times with the assumptions trying to closely represent the reality, during the procedures, many variables may be 


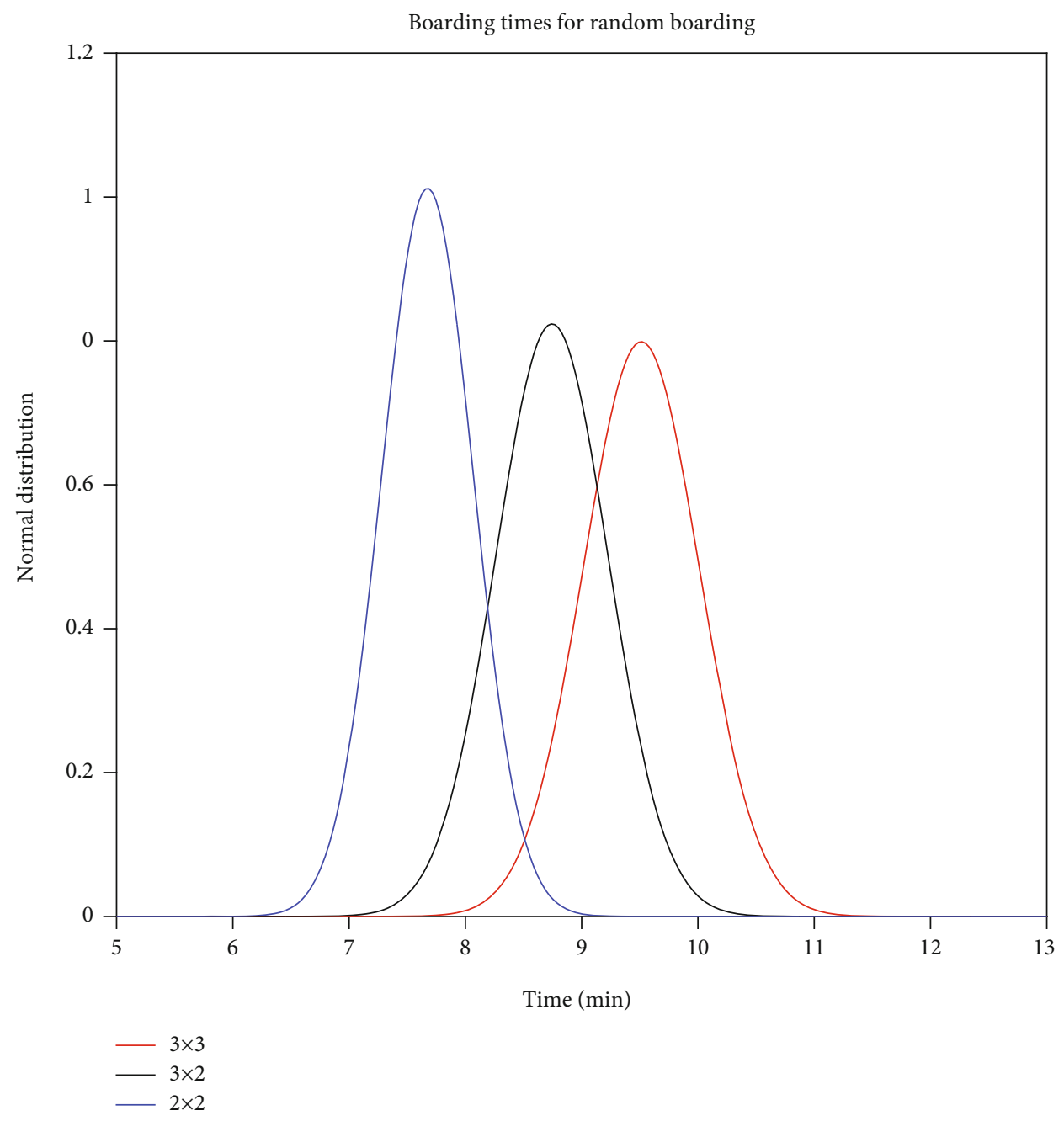

FIGURE 2: Fitted normal distribution curves for the three aircraft configurations using the random boarding strategy.

different and it is hard to predict the behavior of all passengers, especially because, as mentioned before, the model used in this paper is a simplified one the considers that all the passengers are the same with average characteristics. That being said, the boarding and deboarding times represented in this paper may be slightly different from reality. However, the most important part of this paper is the comparison of times amongst the three aircraft configurations. The pattern for time differences and the conclusions on what aircraft configuration has the shortest boarding and deboarding times are more important in this paper than the time itself.

3.2. Boarding Analysis. After the validation process described in Section 3.1, the boarding analyses were carried out using random boarding and random outside-in boarding, as described in Section 2.

For all the analyses, the parameters used for the simulations and assumptions are described as follows:

(i) One carry-on baggage per passenger

(ii) One class for the airplane configuration (iii) Aircraft with 120 passengers

(iv) Passenger action times of 3 seconds to sit down, 3 seconds to get up, and 5 seconds to stow baggage

(v) Aircraft seat configuration of $3 \times 3,3 \times 2$, or $2 \times 2$

(vi) Boarding strategy of random and random outsidein

(vii) Airplane at full capacity (100\% load factor)

In order to assess the influence of aircraft configuration on the boarding time, the first procedure to be analyzed was the random boarding strategy. Figure 2 shows the fitted normal distribution curves for the three aircraft configurations using the random boarding procedure. One can see from that figure that the $2 \times 2$ configuration holds the shortest boarding time, with $3 \times 2$ having the second shortest and $3 \times 3$ holding the longest. Also, configuration $2 \times 2$ holds the smallest standard deviation since it has the highest peak for the fitted normal distribution curve, with configurations 3 $\times 2$ and $3 \times 3$ having almost the same standard deviation. This means that for this boarding strategy, the $2 \times 2$ 
TABLE 2: Comparison of boarding times for the three aircraft configurations using the random boarding strategy.

\begin{tabular}{|c|c|c|c|c|c|}
\hline \multirow[t]{2}{*}{ Configuration } & \multicolumn{2}{|c|}{$\begin{array}{l}\text { Boarding times } \\
\text { (min:sec) }\end{array}$} & \multirow[t]{2}{*}{ STD deviation } & \multirow[t]{2}{*}{ Time reduction (\%) } & \multirow[t]{2}{*}{ Time reduction (min:sec) } \\
\hline & $50 \%$ & $95 \%$ & & & \\
\hline $3 \times 3$ & $9: 31$ & $10: 25$ & $0: 30$ & - & - \\
\hline $3 \times 2$ & $8: 44$ & $9: 33$ & $0: 29$ & $8.23 \% / 8.32 \%$ & $0: 47 / 0: 52$ \\
\hline $2 \times 2$ & $7: 40$ & $8: 19$ & $0: 23$ & $19.44 \% / 20.16 \%$ & 1:51/2:06 \\
\hline
\end{tabular}

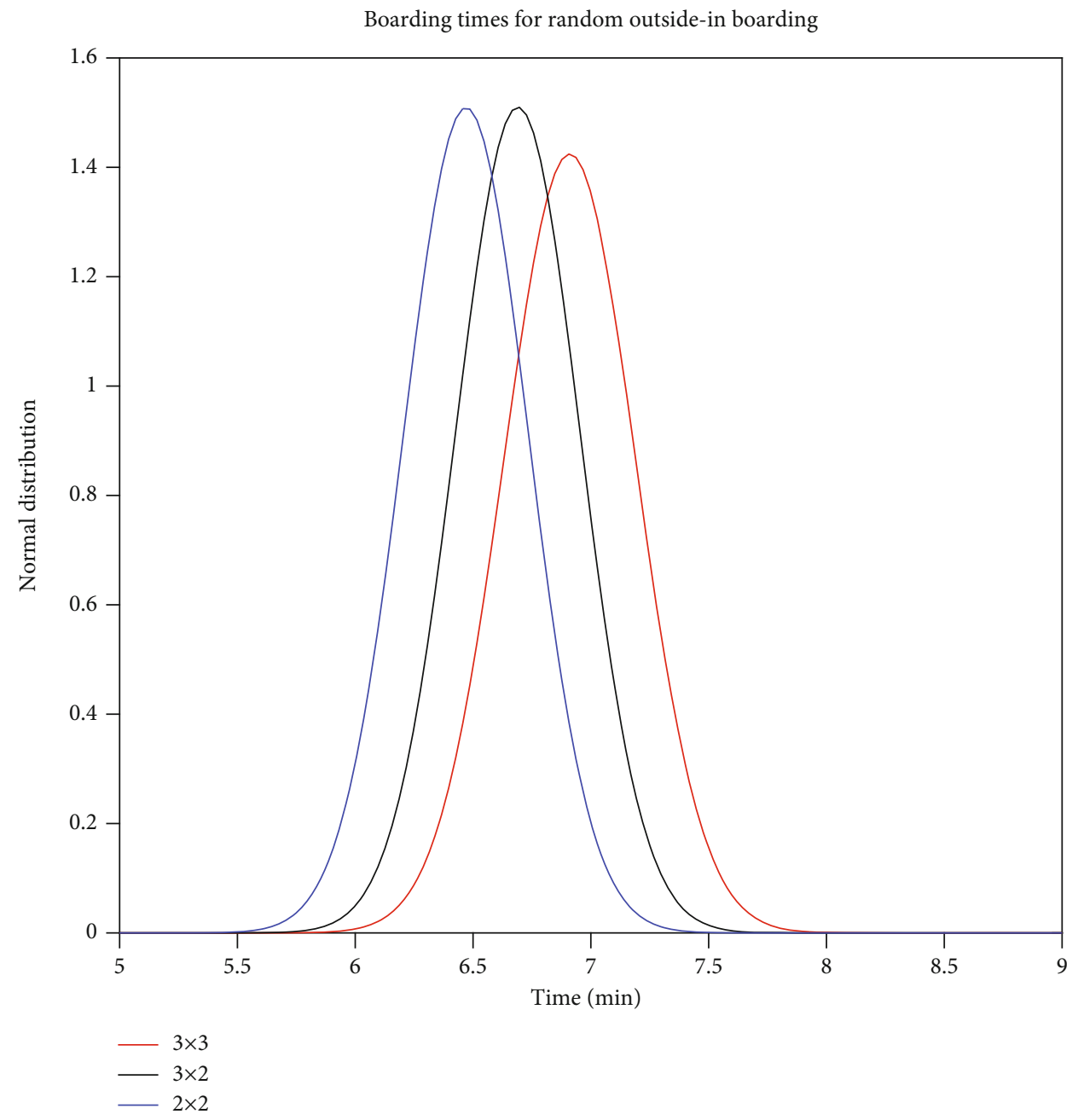

FIGURE 3: Fitted normal distribution curves for the three aircraft configurations using the random outside-in boarding strategy.

configuration is less sensitive to the passenger ordering in the boarding procedure in comparison to the other two, which have similar sensitivity.

For random boarding, configuration $3 \times 3$ has a boarding time of 9:31 min for a 50\% confidence level and 10:25 min for a $95 \%$ confidence level. In comparison to that configuration, the $3 \times 2$ configuration has a time reduction of around $8.32 \%$ and the $2 \times 2$ configuration has a time reduction of $20.16 \%$. The configuration $3 \times 3$ has more passengers to board each row than the other two, which increases its potential for seat and aisle interferences. Since this is a random boarding for all the airplanes, there is a potential for passenger accumulation, which may cause the boarding procedure to take longer.
Table 2 shows the comparison of boarding times for the three configurations using random boarding. The time reduction column represents the reduction in time in comparison to the $3 \times 3$ configuration.

The second procedure to be analyzed was the random outside-in boarding strategy. Figure 3 shows the fitted normal distribution curves for the three aircraft configurations using the random outside-in boarding procedure. Similar to the random boarding, one can see from that figure that the $2 \times 2$ configuration holds the shortest boarding time, with 3 $\times 2$ having the second shortest and $3 \times 3$ holding the longest. Also, configurations $2 \times 2$ and $3 \times 2$ hold almost the same and the smallest standard deviation but with all three having 
TABLE 3: Comparison of boarding times for the three aircraft configurations using the random outside-in boarding strategy.

\begin{tabular}{|c|c|c|c|c|c|}
\hline \multirow[t]{2}{*}{ Configuration } & \multicolumn{2}{|c|}{$\begin{array}{l}\text { Boarding times } \\
\text { (min:sec) }\end{array}$} & \multirow[t]{2}{*}{ STD deviation } & \multirow[t]{2}{*}{ Time reduction (\%) } & \multirow[t]{2}{*}{ Time reduction (min:sec) } \\
\hline & $50 \%$ & $95 \%$ & & & \\
\hline $3 \times 3$ & $6: 54$ & $7: 23$ & $0: 17$ & - & - \\
\hline $3 \times 2$ & $6: 42$ & $7: 06$ & $0: 16$ & $2.90 \% / 3.83 \%$ & $0: 12 / 0: 17$ \\
\hline $2 \times 2$ & $6: 27$ & $6: 57$ & $0: 16$ & $6.52 \% / 5.87 \%$ & $0: 27 / 0: 26$ \\
\hline
\end{tabular}

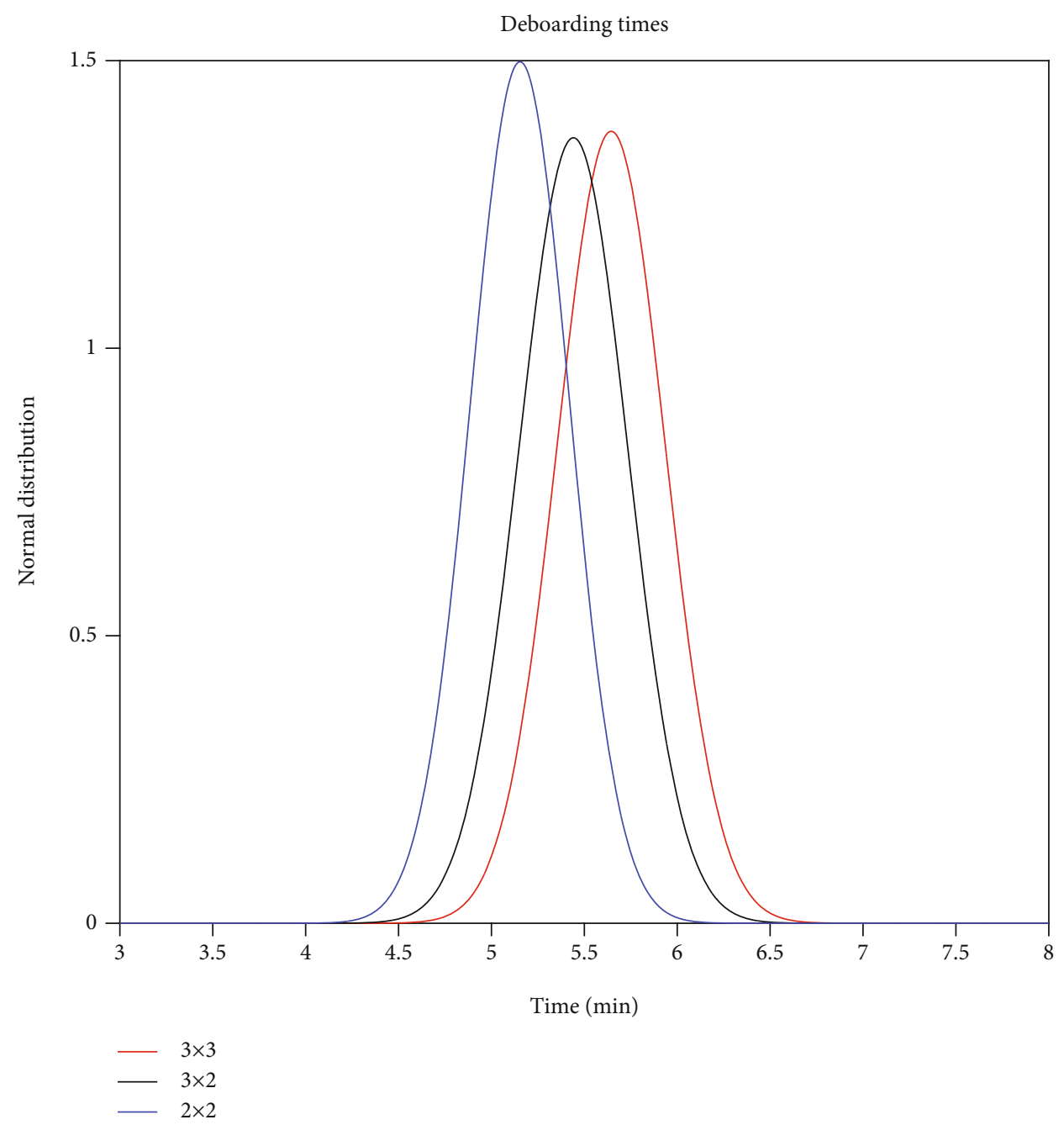

FIGURE 4: Fitted normal distribution curves for deboarding for the three aircraft configurations: $3 \times 3,3 \times 2$, and $2 \times 2$.

very close deviations. This means that for this boarding strategy, all configurations have similar sensitivity to the passenger ordering in the boarding procedure.

For random outside-in boarding, configuration $3 \times 3$ has a boarding time of $6: 54 \mathrm{~min}$ for a $50 \%$ confidence level and $7: 23$ min for a $95 \%$ confidence level. In comparison to that configuration, the $3 \times 2$ configuration has a time reduction of around $3.8 \%$ and the $2 \times 2$ configuration has a time reduction of around $5.9 \%$. One can see from Table 3 that the boarding times for all configurations are close. The time reduction column represents the reduction in time in comparison to the $3 \times 3$ configuration. The outside-in boarding eliminates all seat interferences, leaving only the aisle interferences. Since the boarding times are similar for the three configurations, neither aisle interferences nor aisle length has a great influence on the boarding of single-aisle commercial aircraft. This can only be concluded for the simplified model considering passengers with the same characteristics and the random outside-in boarding. Therefore, seat interferences are the main reason for longer boarding times. This is mainly due to the fact that for each seat interference, the aisle is blocked while all the passengers in the row get up and sit back down. Therefore, seat interferences also cause aisle interferences. 
TABLE 4: Comparison of deboarding times for the three aircraft configurations.

\begin{tabular}{|c|c|c|c|c|c|}
\hline \multirow{2}{*}{ Configuration } & \multicolumn{2}{|c|}{$\begin{array}{l}\text { Deboarding times } \\
\text { (min:sec) }\end{array}$} & \multirow{2}{*}{ STD deviation } & \multirow{2}{*}{ Time reduction (\%) } & \multirow{2}{*}{ Time reduction (min:sec) } \\
\hline & $50 \%$ & $95 \%$ & & & \\
\hline $3 \times 3$ & $5: 39$ & $6: 07$ & $0: 18$ & - & - \\
\hline $3 \times 2$ & $5: 27$ & $5: 55$ & $0: 17$ & $3.54 \% / 3.27 \%$ & $0: 13 / 0: 12$ \\
\hline $2 \times 2$ & 5:09 & $5: 38$ & $0: 16$ & $9.20 \% / 8.01 \%$ & 0:30/0:29 \\
\hline
\end{tabular}

TABLE 5: Comparison of boarding and deboarding times for the three aircraft configurations.

\begin{tabular}{|c|c|c|c|c|c|}
\hline Boarding procedure & Configuration & $B+D$ (min:sec) & $B+D$ reduction $(\%)$ & $B+D$ reduction (min:sec) & Cost savings (US\$/turnaround) \\
\hline \multirow{3}{*}{ Random } & $3 \times 3$ & $16: 32$ & - & - & - \\
\hline & $3 \times 2$ & $15: 28$ & 6.47 & $1: 04$ & 32.10 \\
\hline & $2 \times 2$ & $13: 57$ & 15.66 & $2: 35$ & 77.70 \\
\hline \multirow{3}{*}{ Outside-in } & $3 \times 3$ & $13: 30$ & - & - & - \\
\hline & $3 \times 2$ & 13:01 & 3.56 & $0: 29$ & 14.40 \\
\hline & $2 \times 2$ & $12: 35$ & 6.81 & $0: 55$ & 27.60 \\
\hline
\end{tabular}
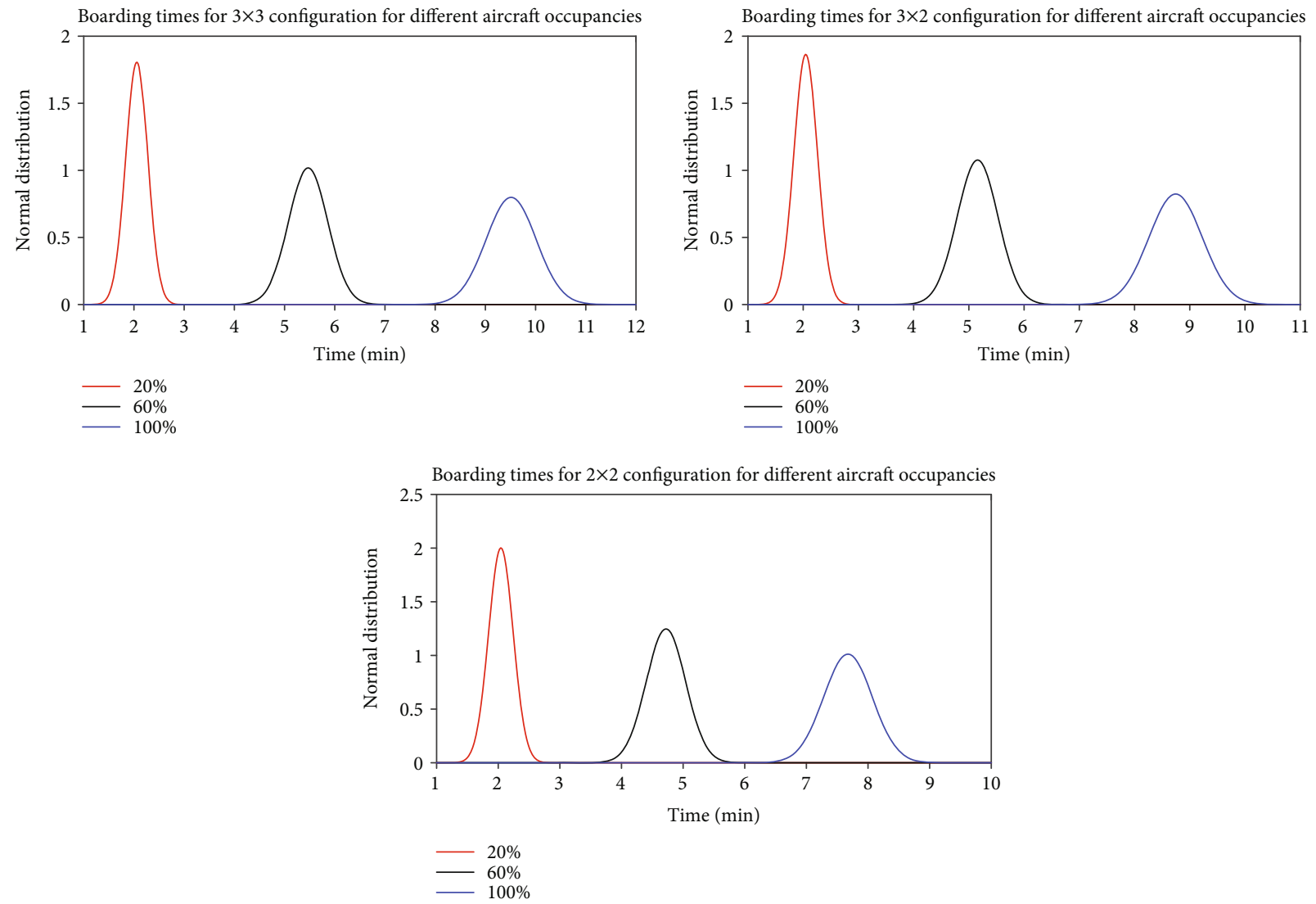

FIGURE 5: Fitted normal distribution curves for the three aircraft configurations using the random boarding strategy for different \% of aircraft occupancy.

3.3. Deboarding Analysis. After all the boarding analyses were performed, the deboarding analyses were carried out. As described in Section 2, differently than the boarding procedure, since this is a simplified model, the deboarding process does not take into account any special queuing or passenger sequence; therefore, only one procedure is considered. As described in Section 3.1, the deboarding tuning parameters are the same as the boarding tuning parameters (time to get 

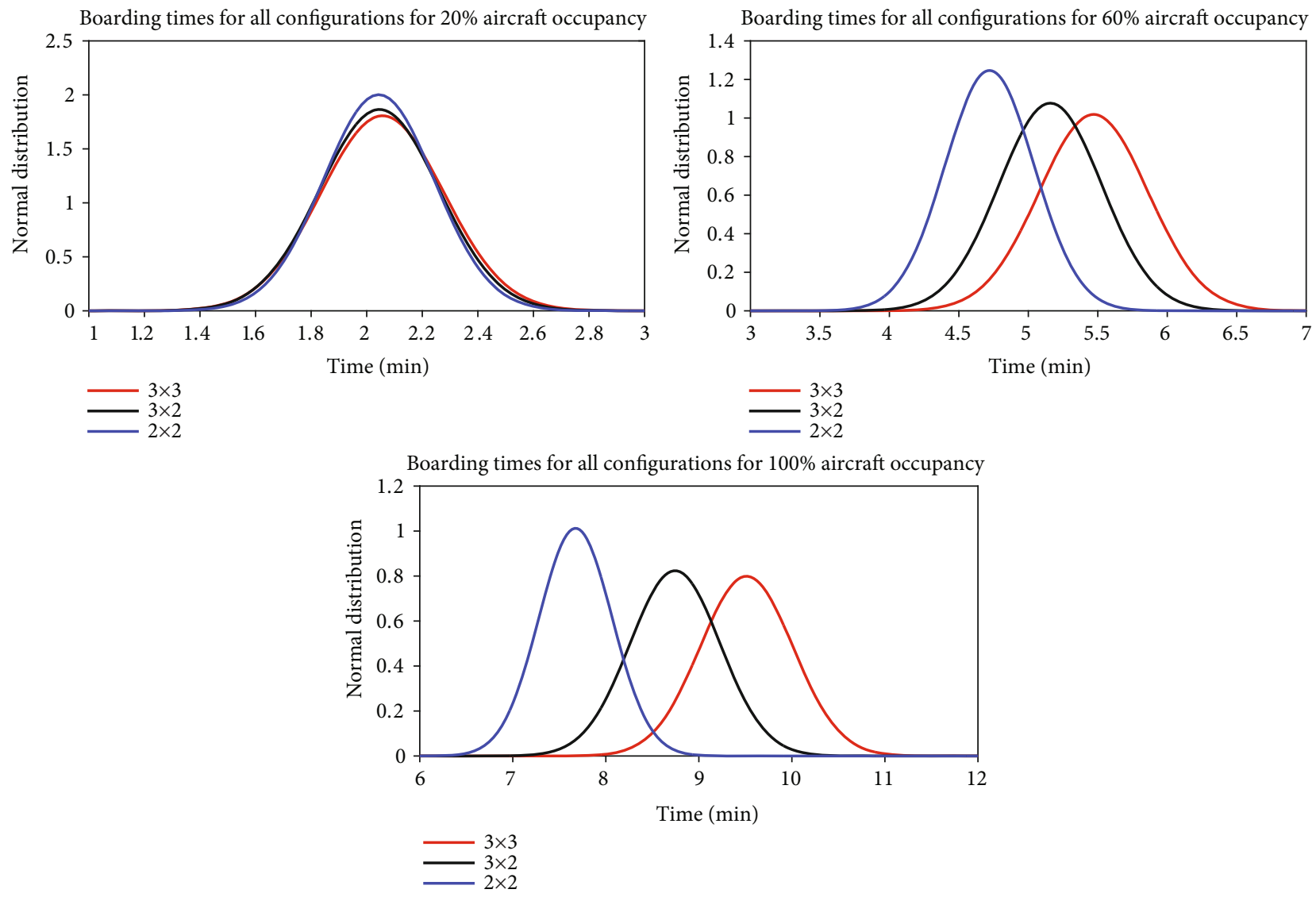

FIGURE 6: Fitted normal distribution curves for each aircraft configuration using the random boarding strategy for different $\%$ of aircraft occupancy.

TABLE 6: Complete comparison of boarding times for the three aircraft configurations using the random boarding strategy for different \% of aircraft occupancy.

\begin{tabular}{|c|c|c|c|c|c|c|c|c|c|c|}
\hline \multirow[b]{2}{*}{ Configuration } & \multicolumn{10}{|c|}{ Times (min:sec) for \% aircraft occupancy } \\
\hline & - & $20 \%$ & $30 \%$ & $40 \%$ & $50 \%$ & $60 \%$ & $70 \%$ & $80 \%$ & $90 \%$ & $100 \%$ \\
\hline \multirow{3}{*}{$3 \times 3$} & $50 \%$ & 2:03 & $2: 52$ & $3: 41$ & $4: 33$ & $5: 27$ & $6: 20$ & $7: 26$ & $8: 28$ & $9: 31$ \\
\hline & $95 \%$ & $2: 26$ & $3: 22$ & $4: 19$ & $5: 14$ & $6: 10$ & $7: 01$ & $8: 13$ & $9: 18$ & $10: 25$ \\
\hline & STD DEV & $0: 13$ & $0: 17$ & $0: 20$ & $0: 22$ & $0: 23$ & $0: 25$ & $0: 28$ & $0: 31$ & $0: 30$ \\
\hline \multirow{3}{*}{$3 \times 2$} & $50 \%$ & 2:01 & $2: 46$ & $3: 32$ & $4: 19$ & 5:08 & $5: 56$ & $6: 51$ & $7: 48$ & $8: 44$ \\
\hline & $95 \%$ & $2: 26$ & $3: 12$ & $4: 02$ & $4: 56$ & $50: 45$ & $6: 39$ & $7: 36$ & $8: 35$ & $9: 33$ \\
\hline & STD DEV & $0: 13$ & $0: 15$ & $0: 18$ & $0: 20$ & $0: 22$ & $0: 25$ & $0: 26$ & $0: 28$ & $0: 29$ \\
\hline \multirow{3}{*}{$2 \times 2$} & $50 \%$ & $2: 03$ & $2: 41$ & $3: 22$ & 4:03 & $4: 42$ & $5: 26$ & $6: 11$ & $6: 56$ & $7: 40$ \\
\hline & $95 \%$ & $2: 23$ & 3:08 & $3: 53$ & $4: 32$ & $5: 15$ & $6: 02$ & $6: 50$ & $7: 37$ & $8: 19$ \\
\hline & STD DEV & $0: 12$ & $0: 15$ & $0: 17$ & $0: 17$ & $0: 19$ & $0: 21$ & $0: 21$ & $0: 23$ & $0: 23$ \\
\hline
\end{tabular}

up and retrieve baggage). For deboarding analysis, the parameters describing the passengers and the aircraft for the simulations are as follows:

(i) One carry-on baggage per passenger

(ii) One class for the airplane configuration

(iii) Aircraft with 120 passengers

(iv) Passenger action times of 3 seconds to get up and 5 seconds to retrieve baggage (v) Aircraft seat configuration of $3 \times 3,3 \times 2$, or $2 \times 2$

(vi) Airplane at full capacity (100\% load factor)

Figure 4 shows the fitted normal distribution curves for the three aircraft configurations for the deboarding procedure. One can see from that figure that the $2 \times 2$ configuration holds the shortest deboarding time, with $3 \times 2$ having the second shortest and $3 \times 3$ holding the longest. Also, configuration $2 \times 2$ holds the smallest standard deviation, with configuration $3 \times 2$ having the second smallest and $3 \times 3$ 

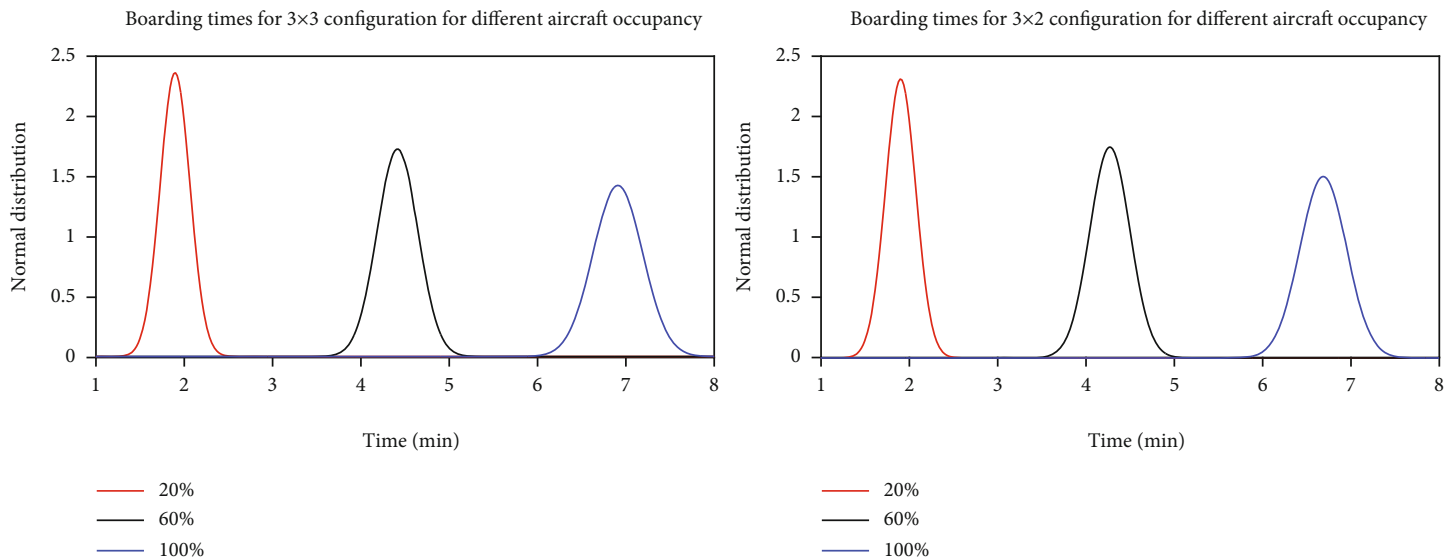

Boarding times for $2 \times 2$ configuration for different aircraft occupancy

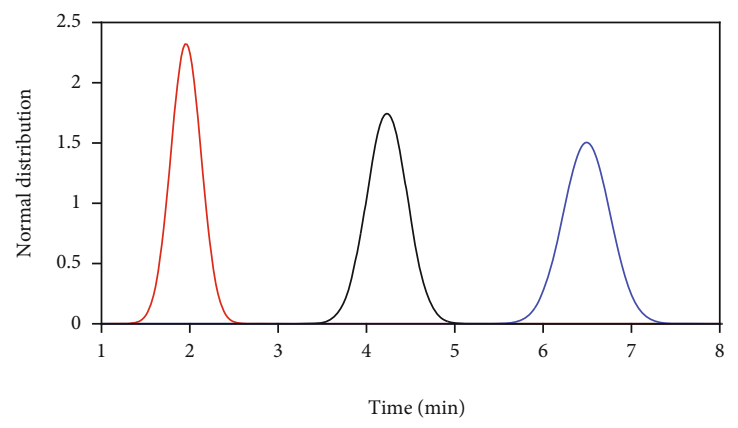

$20 \%$
$-60 \%$

FIGURE 7: Fitted normal distribution curves for the three aircraft configurations using the outside-in boarding strategy for different $\%$ of aircraft occupancy.

having the highest. This means that for deboarding, the $2 \times 2$ configuration is less sensitive to the randomness of the deboarding process in comparison to the other two, which have similar sensitivity.

One can see that the deboarding times shown in Figure 4 are relatively smaller than the boarding times found in Section 3.2 for the random boarding strategy. This is mainly due to the fact that during the deboarding, passengers experience only aisle interferences since, in the same row, the first passenger to get up and leave is the aisle-seated passenger, then the middle-seated, and then the window-seated. Therefore, the deboarding process is similar to the random outsidein boarding in the opposite way. Times for random outsidein boarding are close to deboarding times, which can be seen by comparing Figures 3 and 4 .

Configuration $3 \times 3$ has a deboarding time of 5:39 min for a $50 \%$ confidence level and 6:07 min for a $95 \%$ confidence level. In comparison to that configuration, the $3 \times 2$ configuration has a time reduction of around $3.3 \%$ and the $2 \times 2$ configuration has a time reduction of around $8 \%$. One can see from Table 4 that the deboarding times for all configurations are close. This means that the aircraft configuration does not have a great influence on the deboarding procedure. The time reduction column represents the reduction in time in comparison to the $3 \times 3$ configuration.
3.4. Turnaround Time Analysis. As described in Section 1, boarding and deboarding are part of the critical path of the turnaround time of an airplane in the airport. Airlines are aimed at shortening those times in order to reduce their costs. In this section, the previous separate analyses for boarding and deboarding times were combined in order to perform the comparison for the TAT for the three aircraft configurations. For this analysis, all activities that are part of the TAT (described in Section 1), excluding boarding and deboarding, are considered to be the same independently on the aircraft configuration. Therefore, the comparison for the TAT amongst the three configurations is only dependent on the boarding and deboarding times.

Table 5 shows the sum of boarding and deboarding $(B+D)$ times for the three aircraft configurations, in which all the comparisons were made regarding the $3 \times 3$ configuration for the same boarding strategy. As mentioned, TAT is directly proportional to that sum. Here, all the boarding times used are the $95 \%$ confidence level times described in Sections 3.2 and 3.3. The cost saving comparison (presented here as US\$ per aircraft turnaround procedure) is made using the cost reference based on [6], as described in Section 1, in which the authors suggest that for each minute the airplane is on the ground, it costs US $\$ 30$ to airlines. This analysis is done here for comparison purposes since, in order to have 

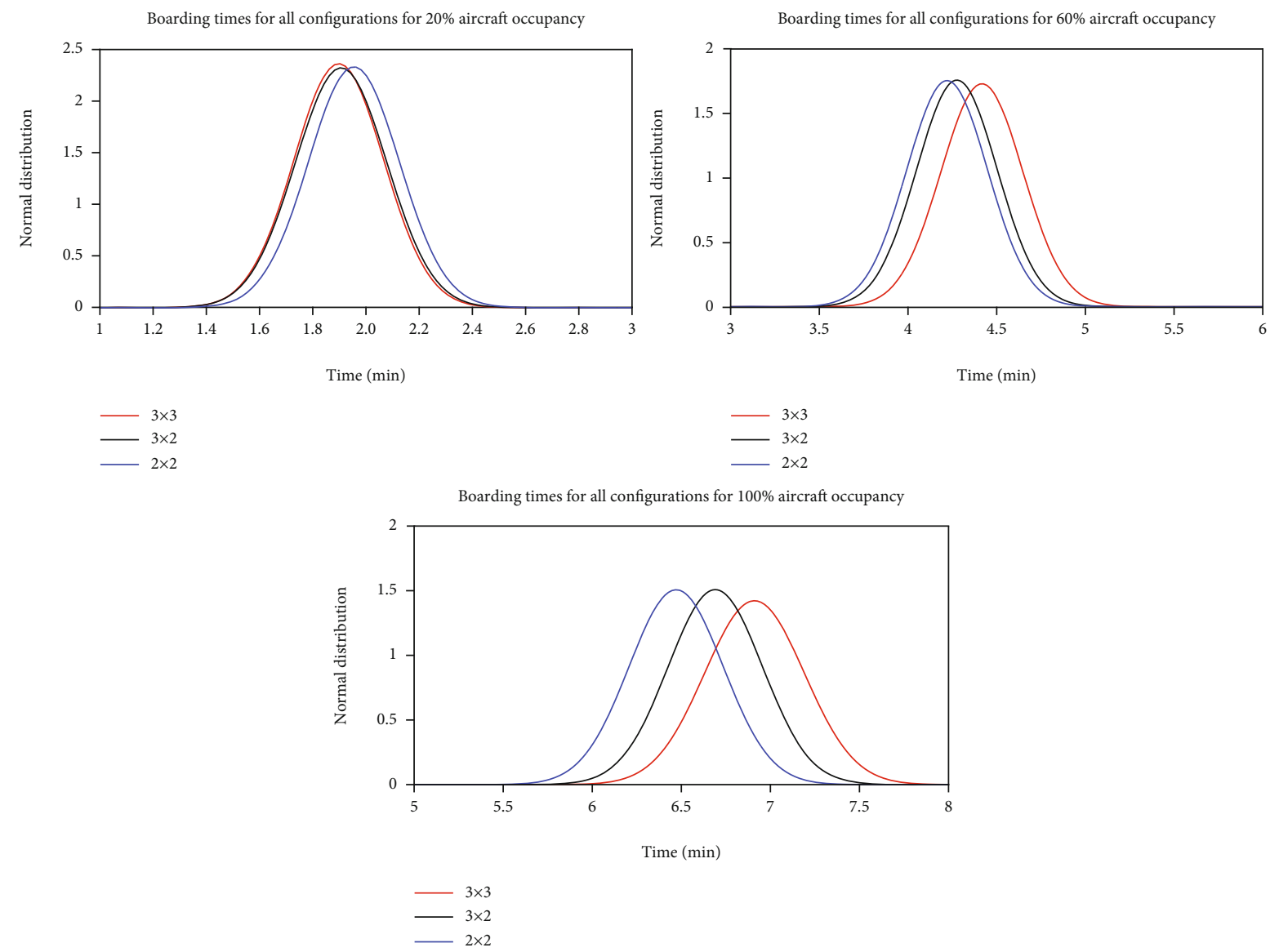

FIGURE 8: Fitted normal distribution curves for each aircraft configuration using the random outside-in boarding strategy for different $\%$ of aircraft occupancy.

those results in real turnaround times, all the assumptions described in Section 2 for boarding and deboarding procedures would have to hold for the airlines, and also other procedures that are part of the TAT would have to be analyzed. Nevertheless, the time reduction comparison is independent of the assumptions made in Section 2 and therefore can be used by airlines as a reference for their cost saving analysis.

For all the procedures used for this analysis, the $2 \times 2$ aircraft configuration holds the shortest TAT and consequently the greatest time reduction and cost savings. The random outside-in procedure holds the smallest time differences due to the fact that seat interferences are eliminated, as described in Section 3.2. This means that if this is the boarding strategy adopted by an airline, the aircraft configuration does not have a great influence on TAT. Nevertheless, random outside-in boarding times are smaller than random boarding times, meaning it has the cheapest TAT.

Since for all the simulations the aircraft have the same number of passengers, the $2 \times 2$ configuration would have longer fuselages or smaller seat pitch. For this paper, it is considered that all aircraft have the same seat pitch, meaning that the $2 \times 2$ aircraft configuration holds the longest fuselage, followed by $3 \times 2$ and then $3 \times 3$. Fuselage size is indirectly evaluated in the simulations in this paper. Even with a longer fuselage, the $2 \times 2$ configuration holds the shortest
TAT, meaning that fuselage size is less relevant for boarding and deboarding than seat and aisle interferences.

\section{Sensitivity Analysis}

All the previous analyses were carried out considering the aircraft is at full capacity (100\% load factor) and all the passengers have carry-on baggage. Nevertheless, it is important to evaluate if the previous results are maintained when those two parameters are varied and also if there is an optimal combination of aircraft configuration and those parameters for the two boarding procedures considered in this paper. For that, a sensitivity analysis was carried out evaluating different aircraft occupancies and different numbers of passengers with carry-on baggage in boarding and deboarding procedures.

4.1. Aircraft Occupancy Influence. The first factor to be analyzed is the aircraft occupancy and its influence on the boarding time using the two aforementioned boarding strategies and the deboarding time for all three configurations. In order to assess the influence of aircraft occupancy, it was varied from $20 \%$ to $100 \%$ occupancy, by steps of $10 \%$. The seats that will have the passengers are assigned randomly, and then passengers are queued according to the boarding procedure. 
TABLE 7: Complete comparison of boarding times for the three aircraft configurations using the random outside-in boarding strategy for different \% of aircraft occupancy.

\begin{tabular}{|c|c|c|c|c|c|c|c|c|c|c|}
\hline \multirow[b]{2}{*}{ Configuration } & \multicolumn{10}{|c|}{ Times (min:sec) for \% aircraft occupancy } \\
\hline & - & $20 \%$ & $30 \%$ & $40 \%$ & $50 \%$ & $60 \%$ & $70 \%$ & $80 \%$ & $90 \%$ & $100 \%$ \\
\hline \multirow{3}{*}{$3 \times 3$} & $50 \%$ & $1: 50$ & $2: 32$ & 3:09 & $3: 47$ & $4: 24$ & 4:59 & $5: 41$ & $6: 19$ & $6: 54$ \\
\hline & $95 \%$ & $2: 11$ & $2: 51$ & $3: 28$ & 4:08 & $4: 48$ & $5: 25$ & $6: 08$ & $6: 46$ & $7: 23$ \\
\hline & STD DEV & $0: 10$ & $0: 11$ & $0: 12$ & $0: 13$ & $0: 14$ & $0: 15$ & $0: 16$ & $0: 16$ & $0: 17$ \\
\hline \multirow{3}{*}{$3 \times 2$} & $50 \%$ & $1: 54$ & $2: 29$ & 3:05 & $3: 42$ & $4: 15$ & $4: 51$ & $5: 30$ & $6: 06$ & $6: 42$ \\
\hline & $95 \%$ & $2: 11$ & $2: 51$ & $3: 26$ & 4:06 & $4: 39$ & $5: 14$ & $5: 55$ & $6: 31$ & $7: 06$ \\
\hline & STD DEV & $0: 10$ & $0: 12$ & $0: 12$ & $0: 13$ & $0: 14$ & $0: 14$ & $0: 16$ & $0: 16$ & $0: 16$ \\
\hline \multirow{3}{*}{$2 \times 2$} & $50 \%$ & $1: 57$ & $2: 32$ & 3:06 & $3: 37$ & $4: 13$ & $4: 43$ & $5: 21$ & $5: 54$ & $6: 27$ \\
\hline & $95 \%$ & $2: 14$ & $2: 52$ & $3: 27$ & $3: 58$ & $4: 37$ & $5: 10$ & $5: 46$ & $6: 23$ & $6: 57$ \\
\hline & STD DEV & $0: 10$ & $0: 12$ & $0: 13$ & $0: 13$ & $0: 14$ & $0: 14$ & $0: 15$ & $0: 16$ & $0: 16$ \\
\hline
\end{tabular}
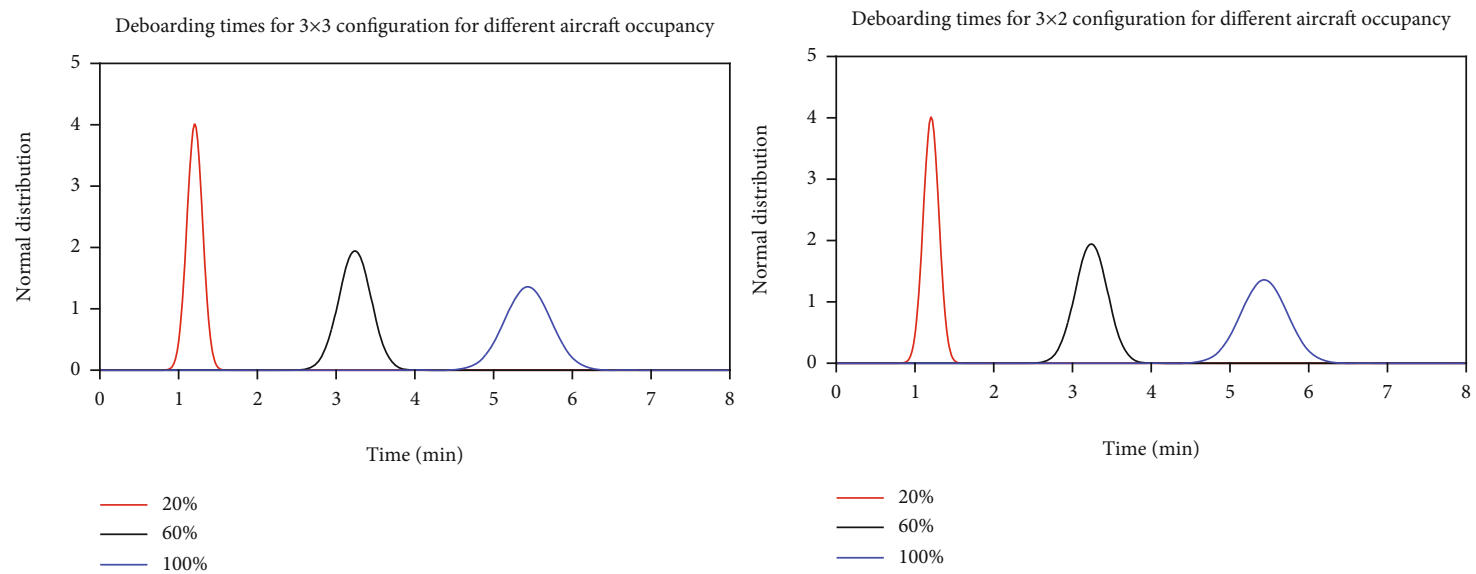

Deboarding times for $2 \times 2$ configuration for different aircraft occupancy

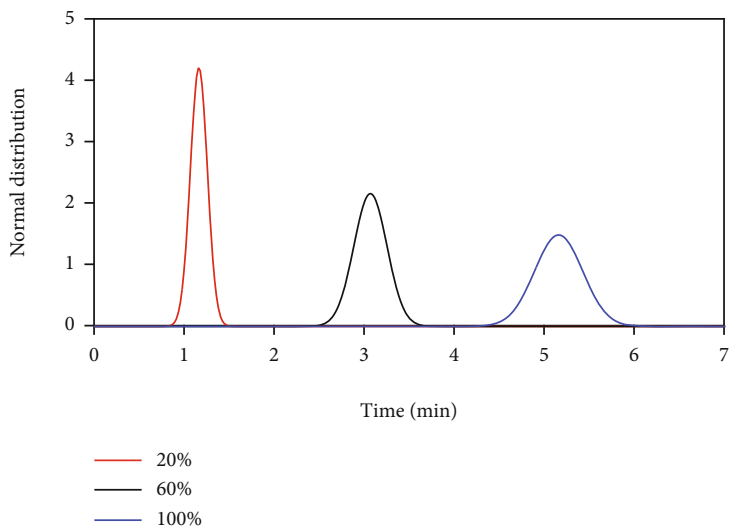

FIGURE 9: Fitted normal distribution curves for deboarding for the three aircraft configurations for different \% of aircraft occupancy.

The parameters and assumptions used for the simulations were as follows:

(i) One carry-on baggage per passenger

(ii) One class for the airplane configuration

(iii) Aircraft with 120 passengers

(iv) Passenger action times of 3 seconds to sit down, 3 seconds to get up, and 5 seconds to stow/retrieve baggage (v) Aircraft seat configuration of $3 \times 3,3 \times 2$, or $2 \times 2$

(vi) Boarding strategy of random and random outsidein

(vii) Airplane capacity from $20 \%$ to $100 \%$ occupancy, by steps of $10 \%$

Figure 5 shows the fitted normal distribution curves for each aircraft configuration using the random boarding procedure for different aircraft occupancies. One can see from Figure 5 that for all configurations, the aircraft occupancy 

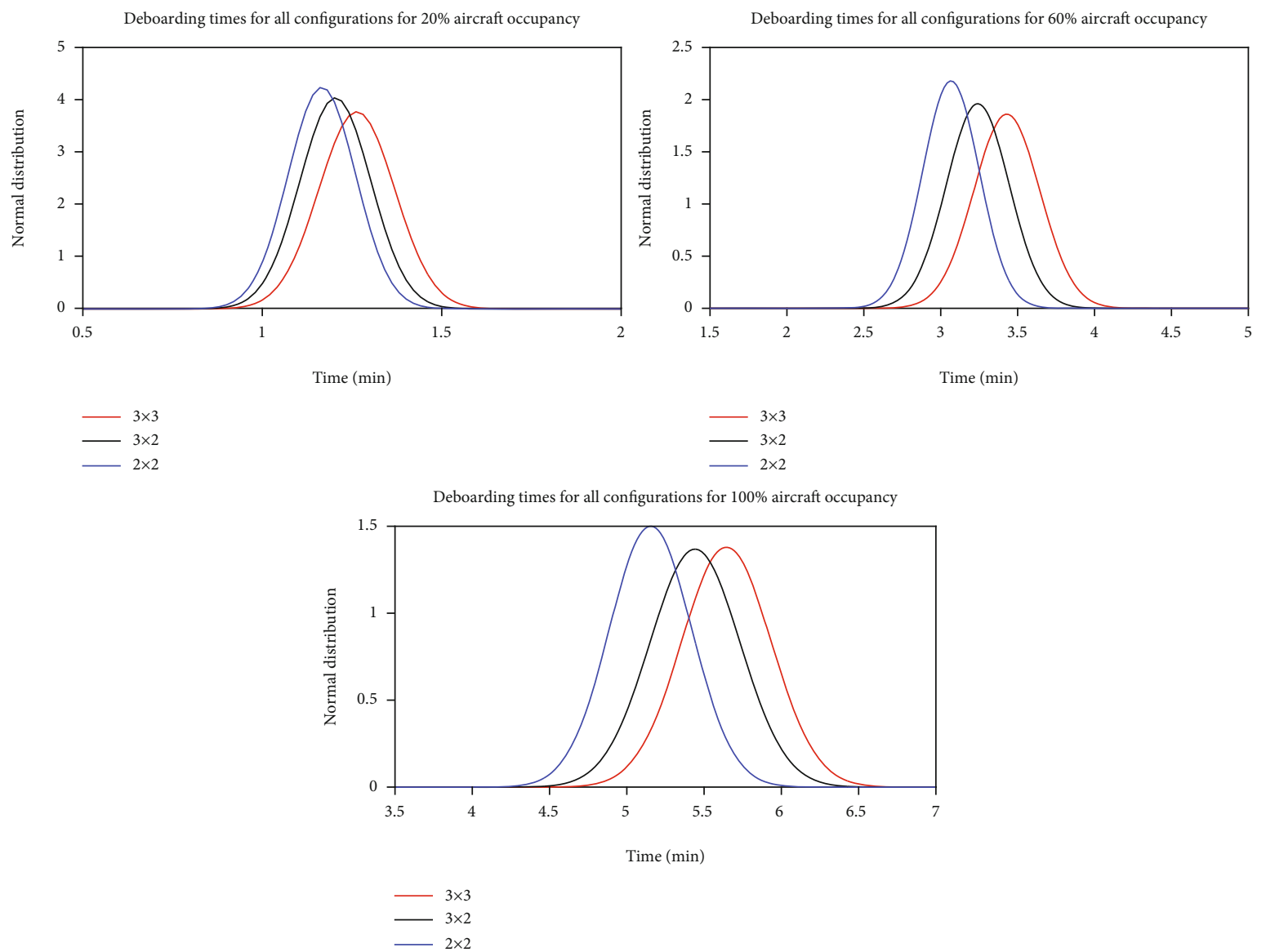

Figure 10: Fitted normal distribution curves for deboarding for each aircraft configuration for different \% of aircraft occupancy.

has an influence on the boarding process since boarding times are shorter for $20 \%$ occupancy than for $100 \%$. Also, the sensitivity to the passenger ordering increases with increasing occupancy since the standard deviation is higher for higher occupancies.

Figure 6 shows the fitted normal distribution curves for each percentage of aircraft occupancy using the random boarding procedure for all aircraft configurations. One can see from Figure 6 that the boarding time for 20\% occupancy is practically the same and that the boarding time difference amongst the three configurations increases with increasing occupancy. Also, the sensitivity to the passenger ordering is similar for all configurations for $20 \%$, and as the occupancy increases, the sensitivity for $3 \times 3$ and $3 \times 2$ configurations tends to be the same, with $2 \times 2$ always having the lower sensitivity.

For random boarding, all configurations have the same boarding time for $20 \%$ occupancy, which is around 2 minutes. Also, with $60 \%$ occupancy, the difference in boarding times for all configurations is not very expressive, and it is considerable for $100 \%$. This indicates that the aircraft configuration has an influence on the boarding time for aircraft occupancies higher than $60 \%$. Table 6 shows the comparison of boarding times for the three configurations using random boarding for different aircraft occupancies.

Figure 7 shows the fitted normal distribution curves for each aircraft configuration using the random outside-in boarding procedure for different aircraft occupancies. Similar to random boarding, for all configurations, the aircraft occupancy has an influence on the boarding process since boarding times are shorter for $20 \%$ occupancy than for $100 \%$. Also, the sensitivity to the passenger ordering increases with increasing occupancy since the standard deviation is higher for higher occupancies.

Figure 8 shows the fitted normal distribution curves for each percentage of aircraft occupancy using the random outside-in boarding procedure for all aircraft configurations. One can see from Figure 8 that the boarding time for $20 \%$ occupancy is practically the same and that the boarding time difference amongst the three configurations increases with increasing occupancy, but the difference in boarding times is not very expressive, similar to what was found in Section 3.2 for the random outside-in boarding. Also, the sensitivity to the passenger ordering is similar for all configurations for all aircraft occupancies, except for $100 \%$ occupancy, in which the sensitivity is higher for $3 \times 3$ configuration and almost the same for $3 \times 2$ and $2 \times 2$ configurations.

For random outside-in boarding, all configurations have practically the same boarding time for $20 \%$ occupancy, which is around 2 minutes. However, this is the only result in which the $2 \times 2$ configuration holds the longer boarding time, which is $1.95 \mathrm{~min}$. This is probably due to the fact that this configuration has a bigger aisle and there is a possibility of a few passengers having their assigned seats in the back of the airplane, 
TABLE 8: Complete comparison of deboarding times for the three aircraft configurations for different \% of aircraft occupancy.

\begin{tabular}{|c|c|c|c|c|c|c|c|c|c|c|}
\hline \multirow[b]{2}{*}{ Configuration } & \multicolumn{10}{|c|}{ Times (min:sec) for \% aircraft occupancy } \\
\hline & - & $20 \%$ & $30 \%$ & $40 \%$ & $50 \%$ & $60 \%$ & $70 \%$ & $80 \%$ & $90 \%$ & $100 \%$ \\
\hline \multirow{3}{*}{$3 \times 3$} & $50 \%$ & $1: 15$ & $1: 46$ & $2: 18$ & $2: 52$ & $3: 26$ & $3: 57$ & $4: 34$ & $5: 08$ & $5: 39$ \\
\hline & $95 \%$ & $1: 26$ & 2:01 & $2: 35$ & $3: 12$ & $3: 48$ & $4: 23$ & $4: 59$ & $5: 35$ & 6:08 \\
\hline & STD DEV & 0:07 & 0:08 & $0: 10$ & $0: 11$ & $0: 13$ & $0: 14$ & $0: 14$ & $0: 16$ & $0: 17$ \\
\hline \multirow{3}{*}{$3 \times 2$} & $50 \%$ & $1: 11$ & $1: 41$ & 2:10 & $2: 41$ & $3: 14$ & $3: 44$ & $4: 21$ & $4: 53$ & $5: 27$ \\
\hline & $95 \%$ & $1: 23$ & $1: 53$ & $2: 26$ & 3:01 & $3: 36$ & $4: 07$ & $4: 48$ & $5: 21$ & $5: 55$ \\
\hline & STD DEV & $0: 06$ & $0: 06$ & 0:09 & $0: 11$ & $0: 12$ & $0: 13$ & $0: 15$ & $0: 16$ & $0: 17$ \\
\hline \multirow{3}{*}{$2 \times 2$} & $50 \%$ & $1: 09$ & $1: 35$ & $2: 03$ & $2: 32$ & 3:04 & $3: 32$ & $4: 05$ & $4: 38$ & 5:08 \\
\hline & $95 \%$ & $1: 21$ & $1: 46$ & $2: 19$ & $2: 50$ & $3: 22$ & $3: 55$ & $4: 29$ & $5: 03$ & $5: 38$ \\
\hline & STD DEV & 0:06 & 0:07 & 0:08 & $0: 10$ & $0: 11$ & $0: 13$ & $0: 14$ & $0: 15$ & $0: 16$ \\
\hline
\end{tabular}
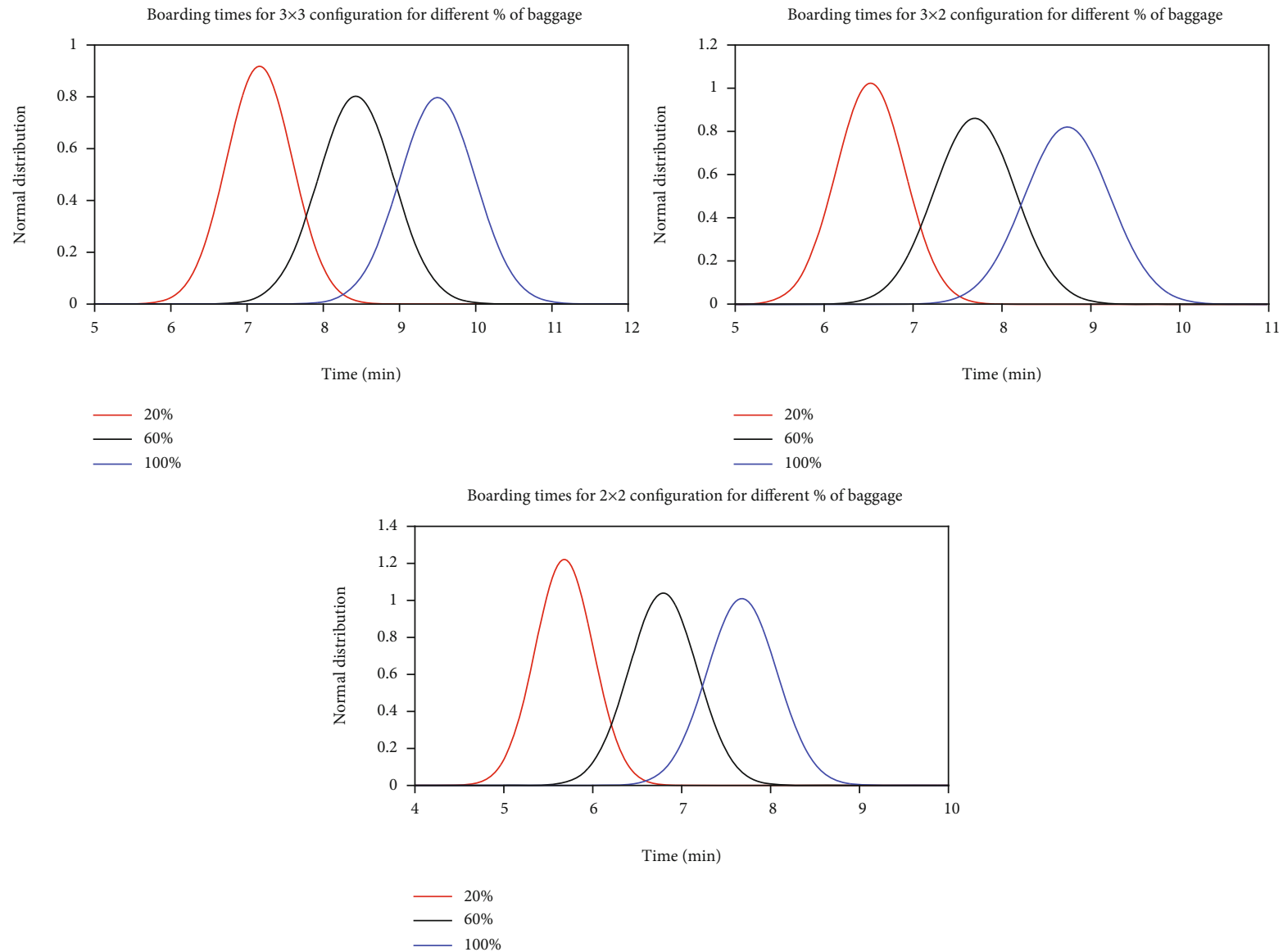

Figure 11: Fitted normal distribution curves for the three aircraft configurations using the random boarding strategy for different \% of baggage.

which will take longer to get to. In this case, the aisle length has more impact on the boarding time than the interferences since both the aisle and seat interferences have a low probability of occurring with $20 \%$ occupancy. Even though there are no models for passengers moving in the aircraft, this conclusion is valid for the simplified model. Table 7 shows the comparison of boarding times for the three configurations using random outside-in boarding for different aircraft occupancies.

Figure 9 shows the fitted normal distribution curves for each aircraft configuration for deboarding for different air- craft occupancies. Similar to the two boarding procedures, for all configurations, the aircraft occupancy has an influence on the deboarding process since deboarding times are shorter for $20 \%$ occupancy than for $100 \%$. Also, the sensitivity to the randomness of the deboarding process increases with increasing occupancy since the standard deviation is higher for higher occupancies.

Figure 10 shows the fitted normal distribution curves for each percentage of aircraft occupancy for the deboarding procedure for all aircraft configurations. One can see from 

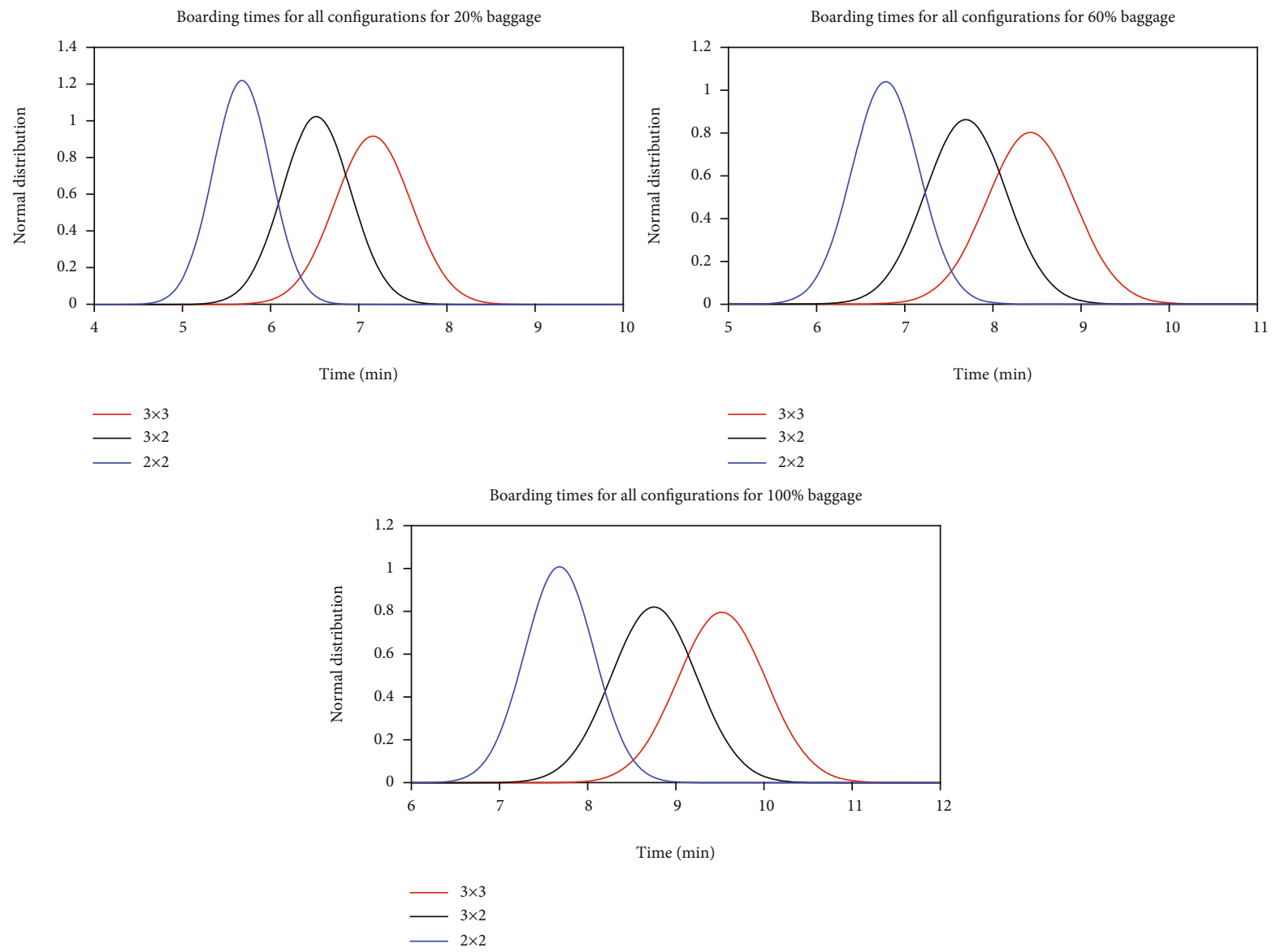

FIGURE 12: Fitted normal distribution curves for each aircraft configuration using the random boarding strategy for different $\%$ of baggage.

Figure 10 that the deboarding time for $20 \%$ occupancy is practically the same for all configurations and that the deboarding time difference amongst the three configurations increases with increasing occupancy. Also, the sensitivity to the randomness of the process for $20 \%$ and $60 \%$ is higher for $3 \times 3$, followed by $3 \times 2$ and $2 \times 2$; for $100 \%$ occupancy, sensitivity is higher for $3 \times 2$, followed by $3 \times 3$ and $2 \times 2$.

For deboarding, for all aircraft occupancies, the difference in deboarding times for all configurations is practically the same. This indicates that for deboarding, the time saving amongst the three configurations is constant and is not dependent on aircraft occupancy. Table 8 shows the comparison of deboarding times for the three configurations for different aircraft occupancies.

4.2. Carry-On Baggage Influence. The second factor to be analyzed is the percentage of passengers having carry-on baggage and its influence on the boarding time using the two aforementioned boarding strategies and the deboarding time for all three configurations. In order to assess the influence of carry-on baggage, the number of passengers with carry-on was varied from $20 \%$ to $100 \%$, by steps of $10 \%$. The seats that will have the passengers with carry-on baggage are assigned randomly, and then passengers are queued according to the boarding procedure. The parameters and assumptions used for the simulations were as follows: (i) One class for the airplane configuration

(ii) Aircraft with 120 passengers

(iii) Passenger action times of 3 seconds to sit down, 3 seconds to get up, and 5 seconds to stow/retrieve baggage

(iv) Aircraft seat configuration of $3 \times 3,3 \times 2$, or $2 \times 2$

(v) Number of passengers with carry-on from $20 \%$ to $100 \%$, by steps of $10 \%$.

(vi) Passengers with carry-on are carrying only one baggage

(vii) Boarding strategy of random and random outside-in

(viii) Airplane capacity of $100 \%$ occupancy

Figure 11 shows the fitted normal distribution curves for each aircraft configuration using the random boarding procedure for different percentages of passengers with carry-on baggage. One can see from Figure 11 that for all configurations, the percentage of passengers with carry-on baggage has an influence on the boarding process since boarding times are shorter for $20 \%$ baggage than for $100 \%$, with $60 \%$ having intermediate times. Also, the sensitivity to the passenger ordering slightly increases with increasing percentages since the standard deviation is higher for higher percentages. 
TABLE 9: Complete comparison of boarding times for the three aircraft configurations using the random boarding strategy for different \% of carry-on baggage.

\begin{tabular}{|c|c|c|c|c|c|c|c|c|c|c|}
\hline \multirow[b]{2}{*}{ Configuration } & \multicolumn{10}{|c|}{ Times (min:sec) for \% carry-on baggage } \\
\hline & - & $20 \%$ & $30 \%$ & $40 \%$ & $50 \%$ & $60 \%$ & $70 \%$ & $80 \%$ & $90 \%$ & $100 \%$ \\
\hline \multirow{3}{*}{$3 \times 3$} & $50 \%$ & $7: 08$ & $7: 29$ & $7: 48$ & $8: 05$ & $8: 25$ & $8: 41$ & $9: 00$ & $9: 14$ & $9: 31$ \\
\hline & $95 \%$ & $7: 55$ & $8: 20$ & $8: 43$ & 8:56 & 9:15 & $9: 31$ & $9: 53$ & 10:09 & $10: 25$ \\
\hline & STD DEV & $0: 26$ & $0: 29$ & $0: 31$ & $0: 30$ & $0: 29$ & $0: 31$ & $0: 32$ & $0: 32$ & $0: 30$ \\
\hline \multirow{3}{*}{$3 \times 2$} & $50 \%$ & $6: 31$ & $6: 51$ & 7:08 & $7: 31$ & $7: 42$ & $7: 56$ & $8: 10$ & $8: 27$ & $8: 44$ \\
\hline & $95 \%$ & 7:09 & $7: 31$ & $7: 54$ & 8:09 & $8: 28$ & $8: 49$ & 9:05 & $9: 14$ & $9: 33$ \\
\hline & STD DEV & $0: 23$ & $0: 25$ & $0: 27$ & $0: 26$ & $0: 28$ & $0: 28$ & $0: 30$ & $0: 28$ & $0: 29$ \\
\hline \multirow{3}{*}{$2 \times 2$} & $50 \%$ & $5: 40$ & $5: 58$ & $6: 15$ & $6: 31$ & $6: 46$ & $7: 03$ & $7: 16$ & $7: 28$ & $7: 40$ \\
\hline & $95 \%$ & $6: 12$ & $6: 35$ & $6: 52$ & $7: 12$ & $7: 27$ & $7: 37$ & $7: 53$ & $8: 13$ & $8: 19$ \\
\hline & STD DEV & $0: 20$ & $0: 20$ & $0: 23$ & $0: 23$ & $0: 23$ & $0: 23$ & $0: 23$ & $0: 24$ & $0: 23$ \\
\hline
\end{tabular}
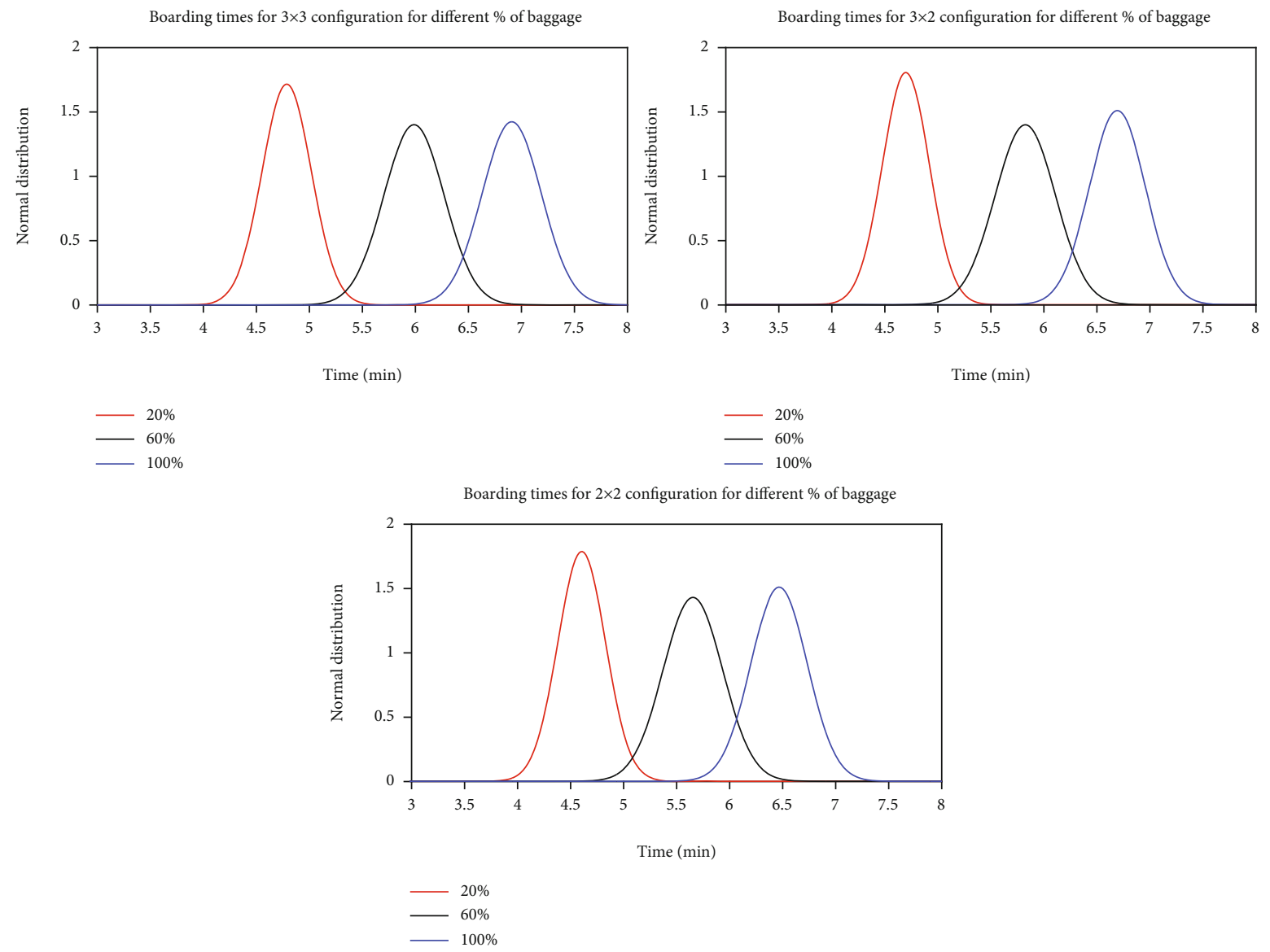

FIGURE 13: Fitted normal distribution curves for the three aircraft configurations using the random outside-in boarding strategy for different $\%$ of baggage.

Figure 12 shows the fitted normal distribution curves for each percentage of passengers with carry-on baggage using the random boarding procedure for all aircraft configurations. One can see from Figure 12 that the difference for boarding time for all percentages of carry-on baggage amongst the three configurations is practically the same. Also, the sensitivity to the passenger ordering has similar behavior for all percentages, with the $3 \times 3$ having the highest sensitivity, followed by $3 \times 2$ and $2 \times 2$.
For random boarding, for all percentages of passengers with carry-on baggage, the difference in boarding times for all configurations is practically the same. This indicates that for random boarding, the time saving for each configuration is constant and is not dependent on the percentage of passengers with carry-on baggage, meaning that there is no optimal combination of aircraft configuration with the percentage of passengers with carry-on baggage. This is due to the fact that in the random boarding procedure, the passengers are 

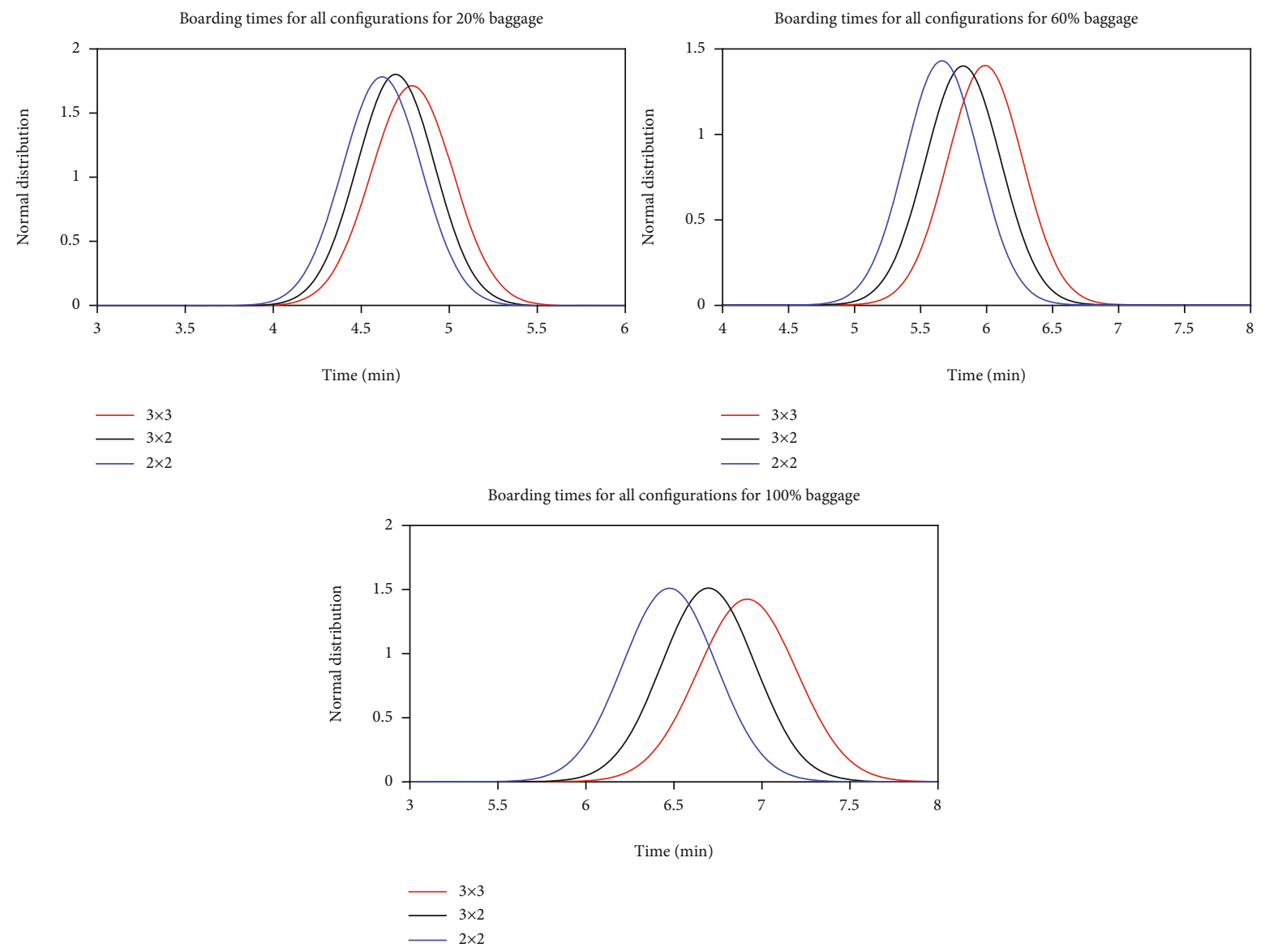

FIGURE 14: Fitted normal distribution curves for each aircraft configuration using the random outside-in boarding strategy for different \% of baggage.

randomly ordered, and the pattern for increasing probability of one passenger interfering with the other in the aisle while stowing the carry-on baggage remains the same for all configurations (the more passengers with carry-on baggage, the more the probability of aisle interferences due to baggage stowing).

The boarding times for each percentage of passengers with carry-on baggage follow the previously observed pattern for random boarding in Section 3.2, with $2 \times 2$ configuration having the shortest boarding times, followed by $3 \times 2$ and then $3 \times 3$. Table 9 shows the comparison of boarding times for the three configurations using random boarding for different percentages of carry-on baggage.

Figure 13 shows the fitted normal distribution curves for each aircraft configuration using the random outside-in boarding procedure for different percentages of passengers with carry-on baggage. One can see from Figure 13 that for all configurations, the percentage of carry-on baggage has an influence on the boarding process since boarding times are shorter for $20 \%$ baggage than for $100 \%$. Different from all previous results, the sensitivity to the passenger ordering is higher for $60 \%$ carry-on baggage than for $100 \%$, meaning that sensitivity to passenger ordering does not increase monotonically.

Figure 14 shows the fitted normal distribution curves for each percentage of passengers with carry-on baggage using the random outside-in boarding procedure for all aircraft configurations. One can see from that figure that the boarding time for $20 \%$ carry-on baggage is practically the same and that the boarding time difference amongst the three configurations increases with increasing percentages of carry-on baggage, but the difference in boarding times is not very expressive, similar to what was found in Sections 3.2 and 4.1. Also, the sensitivity to the passenger ordering is similar for all configurations for all percentages of carry-on baggage, except for $100 \%$ baggage, in which the sensitivity is higher for $3 \times 3$ configuration and almost the same for $3 \times 2$ and $2 \times 2$ configurations.

For random outside-in boarding, the difference in boarding times for all configurations increases with the increase in the percentage of carry-on baggage. This indicates that for random outside-in boarding, the more passengers with carry-on baggage the airplane has, the more efficient the 2 $\times 2$ configuration is in comparison to the other two configurations, but here it is not an expressive time saving.

Differently than random boarding, here, the passengers have a greater probability of interfering with each other due to the concentration in the aisle. This probability increases with the increase in the percentage of passengers with carryon. For the $3 \times 3$ configuration, more passengers have to sit in each row in comparison to the other two configurations, and therefore, the probability of passenger concentration is 
TABLE 10: Complete comparison of boarding times for the three aircraft configurations using the random outside-in boarding strategy for different $\%$ of carry-on baggage.

\begin{tabular}{|c|c|c|c|c|c|c|c|c|c|c|}
\hline \multirow[b]{2}{*}{ Configuration } & \multicolumn{10}{|c|}{ Times (min:sec) for \% carry-on baggage } \\
\hline & - & $20 \%$ & $30 \%$ & $40 \%$ & $50 \%$ & $60 \%$ & $70 \%$ & $80 \%$ & $90 \%$ & $100 \%$ \\
\hline \multirow{3}{*}{$3 \times 3$} & $50 \%$ & $4: 48$ & $5: 09$ & $5: 28$ & $5: 43$ & $6: 00$ & $6: 15$ & $6: 29$ & $6: 42$ & $6: 54$ \\
\hline & $95 \%$ & $5: 10$ & $5: 33$ & $5: 56$ & $6: 10$ & $6: 27$ & $6: 44$ & $6: 56$ & 7:09 & $7: 23$ \\
\hline & STD DEV & $0: 14$ & $0: 16$ & $0: 17$ & $0: 17$ & $0: 17$ & $0: 17$ & $0: 17$ & $0: 17$ & $0: 17$ \\
\hline \multirow{3}{*}{$3 \times 2$} & $50 \%$ & $4: 42$ & $4: 59$ & $5: 17$ & $5: 33$ & $5: 49$ & $6: 04$ & $6: 18$ & $6: 30$ & $6: 42$ \\
\hline & $95 \%$ & 5:04 & $5: 23$ & $5: 45$ & $6: 02$ & $6: 17$ & $6: 31$ & $6: 44$ & $6: 56$ & $7: 06$ \\
\hline & STD DEV & $0: 13$ & $0: 14$ & $0: 15$ & $0: 17$ & $0: 17$ & $0: 17$ & $0: 16$ & $0: 16$ & $0: 16$ \\
\hline \multirow{3}{*}{$2 \times 2$} & $50 \%$ & $4: 37$ & $4: 53$ & $5: 10$ & $5: 26$ & $5: 39$ & $5: 52$ & $6: 05$ & $6: 18$ & $6: 27$ \\
\hline & $95 \%$ & $5: 00$ & $5: 19$ & $5: 34$ & $5: 52$ & $6: 08$ & $6: 21$ & $6: 34$ & $6: 46$ & $6: 57$ \\
\hline & STD DEV & $0: 13$ & $0: 15$ & $0: 15$ & $0: 16$ & $0: 17$ & $0: 17$ & $0: 17$ & $0: 16$ & $0: 16$ \\
\hline
\end{tabular}
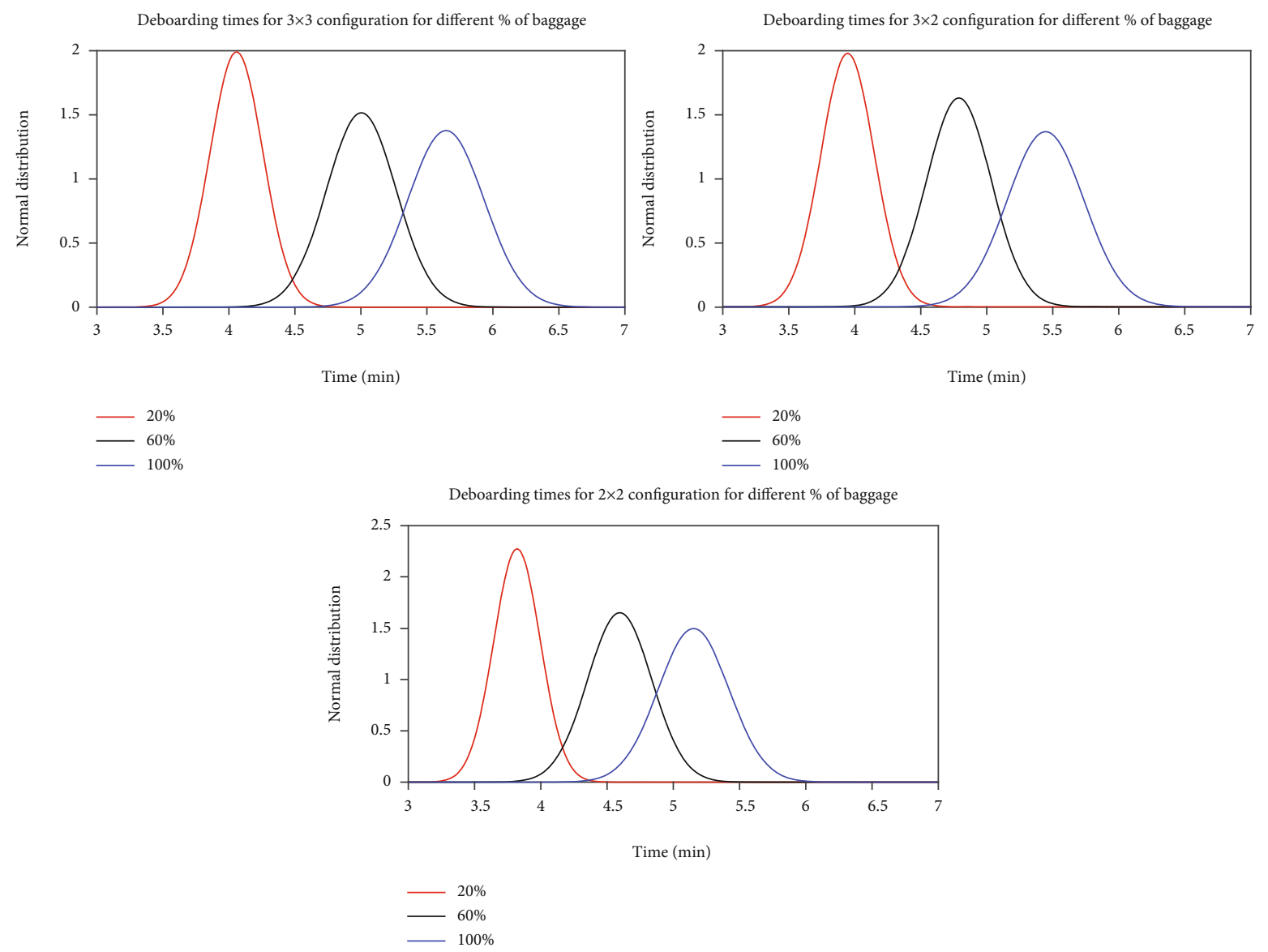

Figure 15: Fitted normal distribution curves for deboarding for the three aircraft configurations for different \% of baggage.

greater. This explains why the difference in boarding times for the three configurations increases with the increase in the number of passengers with carry-on. Table 10 shows the comparison of boarding times for the three configurations using random outside-in boarding for different percentages of carry-on baggage.

Figure 15 shows the fitted normal distribution curves for each aircraft configuration for deboarding for different percentages of passengers with carry-on baggage. One can see from Figure 15 that for all configurations, the percentage of carry-on baggage has an influence on the deboarding process since deboarding times are shorter for $20 \%$ carry-on baggage than for $100 \%$. Also, the sensitivity to the randomness of the deboarding process increases with increasing percentages of carry-on baggage since the standard deviation is higher for higher percentages.

Figure 16 shows the fitted normal distribution curves for each percentage of passengers with carry-on baggage for the deboarding procedure for all aircraft configurations. Similar to random boarding, the difference for deboarding time for 

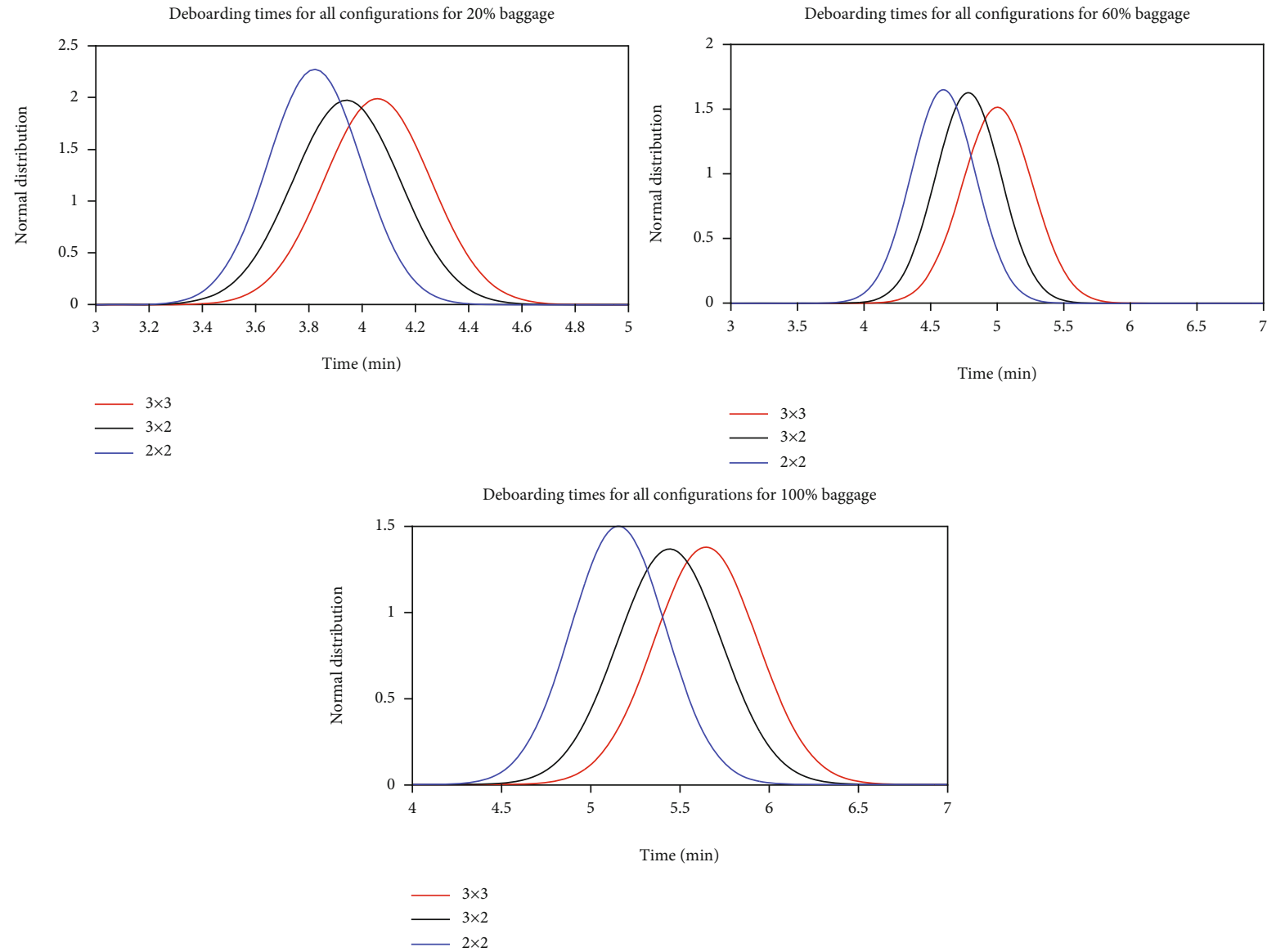

FIGURE 16: Fitted normal distribution curves for deboarding for each aircraft configuration for different \% of baggage.

TABLE 11: Complete comparison of deboarding times for the three aircraft configurations for different \% of carry-on baggage.

\begin{tabular}{|c|c|c|c|c|c|c|c|c|c|c|}
\hline \multirow[b]{2}{*}{ Configuration } & \multicolumn{10}{|c|}{ Times (min:sec) for \% carry-on baggage } \\
\hline & - & $20 \%$ & $30 \%$ & $40 \%$ & $50 \%$ & $60 \%$ & $70 \%$ & $80 \%$ & $90 \%$ & $100 \%$ \\
\hline \multirow{3}{*}{$3 \times 3$} & $50 \%$ & 4:03 & $4: 18$ & $4: 32$ & $4: 47$ & $5: 00$ & $5: 11$ & $5: 22$ & $5: 32$ & $5: 39$ \\
\hline & $95 \%$ & $4: 26$ & $4: 44$ & $4: 58$ & $5: 13$ & $5: 26$ & $5: 39$ & $5: 50$ & $6: 00$ & $6: 07$ \\
\hline & STD DEV & $0: 12$ & $0: 14$ & $0: 14$ & $0: 16$ & $0: 16$ & $0: 17$ & $0: 17$ & $0: 17$ & $0: 17$ \\
\hline \multirow{3}{*}{$3 \times 2$} & $50 \%$ & $3: 55$ & $4: 11$ & $4: 25$ & $4: 36$ & $4: 46$ & $4: 57$ & $5: 07$ & $5: 18$ & $5: 27$ \\
\hline & $95 \%$ & $4: 17$ & $4: 32$ & $4: 52$ & $5: 01$ & $5: 14$ & $5: 27$ & $5: 34$ & $5: 46$ & $5: 55$ \\
\hline & STD DEV & $0: 12$ & $0: 12$ & $0: 14$ & $0: 14$ & $0: 15$ & $0: 16$ & $0: 16$ & $0: 17$ & $0: 17$ \\
\hline \multirow{3}{*}{$2 \times 2$} & $50 \%$ & $3: 48$ & $4: 02$ & $4: 14$ & $4: 25$ & $4: 35$ & $4: 44$ & $4: 55$ & $5: 04$ & $5: 08$ \\
\hline & $95 \%$ & $4: 07$ & $4: 23$ & $4: 34$ & $4: 50$ & $5: 02$ & $5: 10$ & $5: 19$ & $5: 31$ & $5: 38$ \\
\hline & STD DEV & $0: 11$ & $0: 11$ & $0: 13$ & $0: 14$ & $0: 14$ & $0: 14$ & $0: 16$ & $0: 17$ & $0: 16$ \\
\hline
\end{tabular}

all percentages of carry-on baggage amongst the three configurations is practically the same. The sensitivity to the randomness of the process has similar behavior for $20 \%$ and $100 \%$, with the $3 \times 2$ having the highest sensitivity, followed by $3 \times 3$ and $2 \times 2$; for $60 \%$, configurations $3 \times 2$ and $2 \times 2$ have similar sensitivity, with $3 \times 3$ having the highest.

For deboarding, the difference in deboarding times for all configurations is similar to the increase in the percentage of carry-on baggage. This indicates that for deboarding, the amount of passengers with carry-on does not change which configuration has the best potential to cost savings.
Table 11 shows the comparison of deboarding times for the three configurations for different percentages of carry-on baggage.

\section{Conclusions}

This paper presented the analysis of the influence of commercial aircraft interior configuration on the boarding and deboarding procedures. Computer simulation was used to assess boarding and deboarding times, considering two boarding strategies: random boarding and random outside- 
in boarding. The tool used for the simulation was developed in MATLAB ${ }^{\mathrm{TM}}$ using object-oriented programming. All the analyses have statistical treatment in order to make comparisons more feasible. Three aircraft configurations, all with 120 passengers, were used for the comparisons: single aisle $3 \times 3$ ("six abreast"), single aisle $3 \times 2$ ("five abreast"), and single aisle $2 \times 2$ ("four abreast"). After the analysis of the influence of interior configuration, a sensitivity analysis was performed to evaluate the influence of two factors on the results: the aircraft occupancy and the number of passengers with carry-on luggage.

For all boarding procedures, the $2 \times 2$ interior configuration is the one with the shortest times, followed by $3 \times 2$ and $3 \times 3$. The $2 \times 2$ configuration has less probability of having seat interferences per row since it has only two passengers per row on both sides of the aircraft.

For the random outside-in boarding, the boarding times for all configurations are close, and this is the boarding strategy that holds shorter boarding times in comparison to random boarding. The outside-in boarding eliminates all seat interferences by boarding the passengers from the window seat to the aisle seat, leaving only aisle interferences to cause disturbances in the process. This result is consistent with what is found in the literature. Nevertheless, studies state that this method is difficult to implement since it may cause groups that travel together (and usually sit in the same row) to board the airplane in different moments, which may cause passenger dissatisfaction and also passenger confusion during the boarding process. Since seat interferences are eliminated and the boarding time is similar to the three aircraft configurations, for the boarding procedures evaluated, it can be concluded that aisle interferences do not exert a great influence on the boarding of single-aisle commercial aircraft, leaving seat interferences to be the main reason for longer boarding times.

Different from passenger boarding, the deboarding times for all configurations are very similar, indicating the aircraft configuration does not exert a great influence on the deboarding process. Deboarding is accomplished by each passenger occupying empty spaces in the aircraft, meaning that passengers can execute actions simultaneously in the whole aircraft, decreasing the influence of the aircraft configuration on this procedure. However, the $2 \times 2$ configuration has a slight advantage in comparison to the other two.

Since the $2 \times 2$ is the one that holds the shortest boarding times and deboarding times are similar amongst the configurations, the $2 \times 2$ configuration is the one with the shortest TAT and consequently the greatest potential for cost savings for airlines. In order to have those results in real turnaround times, all the assumptions described in this paper for boarding and deboarding procedures would have to hold for the airlines, and also other procedures that are part of the TAT would have to be analyzed to assess if they do not become part of the critical path after boarding time reduction.

For the two boarding procedures evaluated and for deboarding, the results indicate that aircraft occupancy exerts an influence on the boarding and deboarding times for all configurations. For random boarding, the aircraft configura- tion exerts an influence on the boarding time only for aircraft occupancies higher than $60 \%$. For all aircraft occupancies in all boarding strategies evaluated, the $2 \times 2$ configuration is still the one that holds the shortest boarding and deboarding times. The only exception is in the random outside-in boarding with $20 \%$ occupancy in which the $2 \times 2$ configuration holds the longer boarding time. This is probably due to the fact that this configuration has the biggest aisle and there is a possibility of a few passengers having their assigned seats in the back of the airplane, which will take longer for them to get to. This situation does not happen in all other analyses because of the effect of aisle and seat interferences, which is less likely to happen with $20 \%$ occupancy. Even though there are no models for passengers moving in the aircraft, this conclusion is valid for the simplified model.

For the two boarding procedures and for deboarding, the results indicate that the percentage of passengers with carryon baggage exerts an influence on the boarding and deboarding times for all configurations. For all carry-on baggage percentages in all boarding strategies, the $2 \times 2$ configuration is still the one that holds the shortest boarding and deboarding times. For random outside-in boarding, the more passengers with carry-on baggage the airplane has, the more efficient the $2 \times 2$ configuration is in comparison to the other two configurations, but here it is not an expressive time saving. For random boarding, the behavior of time reduction of $2 \times 2$ in comparison to the other two configurations is constant for all baggage percentages.

Therefore, based on the results and discussions, the aircraft interior configuration exerts an influence on the boarding and deboarding times. Also, the aircraft occupancy and number of passengers with carry-on baggage influence the results.

An interesting approach with this model is to use it as a constraint for a Multidisciplinary Design Optimization (MDO). When designing an aircraft, manufacturers perform several trade-offs with a wide range of parameters. A reduced TAT can be one of those parameters, and a fair interior configuration can be achieved when this parameter is part of the MDO process performed by those manufacturers. For future work, enhancements to the model should allow the following studies:

(i) For the deboarding process, the model could be used to simulate emergency evacuation procedures using multiple doors and emergency exits over the wing

(ii) A model for passengers walking inside the aircraft could be implemented

(iii) An MDO process can be performed using this model and other parameters to perform a preliminary interior configuration design of an aircraft

\section{Data Availability}

Data can be available if the paper is published upon request to the corresponding author. 


\section{Conflicts of Interest}

The authors declare that they have no conflict of interest.

\section{Authors' Contributions}

All authors contributed to the study conception and design. Material preparation and analysis were performed by Diogo Bertolini Profeta de Oliveira, Alison de Oliveira Moraes, and Jonathan Naves Coelho. The first draft of the manuscript was written by Diogo Bertolini Profeta de Oliveira, and all authors commented on previous versions of the manuscript. All authors read and approved the final manuscript.

\section{Acknowledgments}

DBPO and JNC thank the support provided by the Engineering Specialization Program (PEE).

\section{References}

[1] C. L. Wu, Airline Operations and Delay Management: Insights from Airline Economics, Networks, and Strategic Schedule Planning, Ashgate Publishing Limited, England, 1st edition, 2010.

[2] F. Jaehn and S. Neumann, "Airplane boarding," European Journal of Operational Research, vol. 244, no. 2, pp. 339-359, 2015.

[3] H. van Landeghem and A. Beuselinck, "Reducing passenger boarding time in airplanes: a simulation based approach," European Journal of Operational Research, vol. 142, no. 2, pp. 294-308, 2002.

[4] M. Bazargan, "A linear programming approach for aircraft boarding strategy," European Journal of Operational Research, vol. 183, no. 1, pp. 394-411, 2007.

[5] A. Steiner and M. Philipp, "Speeding up the airplane boarding process by using pre-boarding areas," in Swiss Transport Research Conference, Ascona, 2009.

[6] D. C. Nyquist and K. L. McFadden, "A study of the airline boarding problem," Journal of the Air Transport Management, vol. 14, no. 4, pp. 197-204, 2008.

[7] M. Schmidt, "A review of aircraft turnaround operations and simulations," Progress Aerospace Sciences, vol. 92, pp. 25-38, 2017.

[8] J. H. Steffen, "Optimal boarding method for airline passengers," Journal of the Air Transport Management, vol. 14, no. 3, pp. 146-150, 2008.

[9] R. J. Milne and A. R. Kelly, "A new method for boarding passengers onto an airplane," Journal of the Air Transport Management, vol. 34, pp. 93-100, 2014.

[10] P. Ferrari and K. Nagel, "Robustness of efficient passenger boarding strategies for airplanes," Journal of the Transportation Research Board, vol. 1915, no. 1, pp. 44-54, 2005.

[11] H. Zeineddine, "A dynamically optimized aircraft boarding strategy," Journal of the Air Transport Management, vol. 58, pp. 144-151, 2017.
[12] A. Kierzkowski and T. Kisiel, "The human factor in the passenger boarding process at the airport," in International Scientific Conference Transbaltica 2017: Transportation Science and Technology, pp. 348-355, Vilnius Gediminas Technical University, Vilnius, Lithuania, 2017.

[13] M. Schultz, "Fast aircraft turnaround enabled by reliable passenger boarding," Aerospace, vol. 5, no. 1, p. 8, 2018. 